PAPER

\section{Spin-glass dynamics in the presence of a magnetic field: exploration of microscopic properties}

To cite this article: I Paga et al J. Stat. Mech. (2021) 033301

View the article online for updates and enhancements.
You may also like

- $\frac{\text { Research on Ammunition Simulation }}{\text { Method }}$
$\frac{\text { Sun Yong, Li Jun and Fan Kaijun }}{\text { - Performance Simulation of Tubular }}$
$\frac{\text { Segmented-in-Series SOFC Using }}{\text { Simplified Equivalent Circuit }}$
Shun Yoshida, Tadashi Tanaka and
Yoshitaka Inui
- (Invited) Multiscale Modeling of Atom
Scale Defects for Electronic Devices
Enqineering
Luca Larcher, Federico Nardi, Dipankar
Pramanik et al.

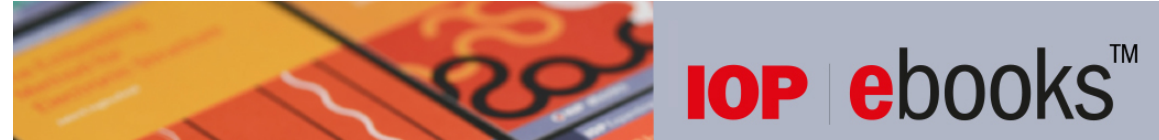

Bringing together innovative digital publishing with leading authors from the global scientific community. Start exploring the collection-download the first chapter of every title for free. 


\section{Spin-glass dynamics in the presence of a magnetic field: exploration of microscopic properties}

I Paga ${ }^{1,2,20, *}$, Q Zhai ${ }^{3,20}$, M Baity-Jesi ${ }^{4}$, E Calore ${ }^{5}$, A Cruz ${ }^{6,7}$, L A Fernandez 2,7 , J M Gil-Narvion', I Gonzalez-Adalid Pemartin ${ }^{2}$, A Gordillo-Guerrero ${ }^{7,8,9}$, D Iñiguez ${ }^{6,7,10}$, A Maiorano ${ }^{7,11,12}$, E Marinari ${ }^{12,13}$, V Martin-Mayor ${ }^{2,7}$, J Moreno-Gordo ${ }^{6,7}$, A Muñoz-Sudupe ${ }^{2,7}$, D Navarro ${ }^{14}$, R L Orbach ${ }^{3}$, G Parisi $^{12,13}$, S Perez-Gaviro ${ }^{6,7,15}$, F Ricci-Tersenghi ${ }^{12,13}$, J J Ruiz-Lorenzo ${ }^{7,9,16}$, S F Schifano ${ }^{17}$, D L Schlagel ${ }^{18}$, B Seoane $^{2,7}$, A Tarancon ${ }^{6,7}, \mathbf{R}$ Tripiccione $^{5}$ and D Yllanes ${ }^{7,19}$

1 Dipartimento di Fisica, Sapienza Università di Roma, INFN, Sezione di Roma 1, I-00185 Rome, Italy

2 Departamento de Física Teórica, Universidad Complutense, 28040 Madrid, Spain

3 Texas Materials Institute, The University of Texas at Austin, Austin, TX 78712, United States of America

4 Eawag, Überlandstrasse 133, CH-8600 Dübendorf, Switzerland

5 Dipartimento di Fisica e Scienze della Terra, Università di Ferrara e INFN, Sezione di Ferrara, I-44122 Ferrara, Italy

6 Departamento de Física Teórica, Universidad de Zaragoza, 50009 Zaragoza, Spain

7 Instituto de Biocomputación y Física de Sistemas Complejos (BIFI), 50018 Zaragoza, Spain

8 Departamento de Ingeniería Eléctrica, Electrónica y Automática, U. de Extremadura, 10003, Cáceres, Spain

9 Instituto de Computación Científica Avanzada (ICCAEx), Universidad de Extremadura, 06006 Badajoz, Spain

10 Fundación ARAID, Diputación General de Aragón, 50018 Zaragoza, Spain 
Spin-glass dynamics in the presence of a magnetic field: exploration of microscopic properties

11 Dipartimento di Biotecnologie, Chimica e Farmacia, Università degli

Studi di Siena, 53100, Siena, Italy

12 Dipartimento di Fisica, Sapienza Università di Roma, and CNR-Nanotec, I-00185 Rome, Italy

${ }^{13}$ INFN, Sezione di Roma 1, I-00185 Rome, Italy

${ }^{14}$ Departamento de Ingeniería, Electrónica y Comunicaciones and I3A,

U. de Zaragoza, 50018 Zaragoza, Spain

${ }^{15}$ Centro Universitario de la Defensa, 50090 Zaragoza, Spain

16 Departamento de Física, Universidad de Extremadura, 06006 Badajoz, Spain

17 Dipartimento di Scienze Chimiche e Farmaceutiche, Università di Ferrara

e INFN Sezione di Ferrara, I-44122 Ferrara, Italy

18 Division of Materials Science and Engineering, Ames Laboratory, Ames,

IA 50011, United States of America

${ }^{19}$ Chan Zuckerberg Biohub, San Francisco, CA 94158, United States of

America

E-mail: ilaria.paga@uniroma1.it

Received 4 January 2021

Accepted for publication 8 January 2021

Published 8 March 2021

Online at stacks.iop.org/JSTAT/2021/033301

https://doi.org/10.1088/1742-5468/abdfca

Abstract. The synergy between experiment, theory, and simulations enables a microscopic analysis of spin-glass dynamics in a magnetic field in the vicinity of and below the spin-glass transition temperature $T_{\mathrm{g}}$. The spin-glass correlation length, $\xi\left(t, t_{\mathrm{w}} ; T\right)$, is analysed both in experiments and in simulations in terms of the waiting time $t_{\mathrm{w}}$ after the spin glass has been cooled down to a stabilised measuring temperature $T<T_{\mathrm{g}}$ and of the time $t$ after the magnetic field is changed. This correlation length is extracted experimentally for a CuMn 6 at. $\%$ single crystal, as well as for simulations on the Janus II special-purpose supercomputer, the latter with time and length scales comparable to experiment. The non-linear magnetic susceptibility is reported from experiment and simulations, using $\xi\left(t, t_{\mathrm{w}} ; T\right)$ as the scaling variable. Previous experiments are reanalysed, and disagreements about the nature of the Zeeman energy are resolved. The growth of the spin-glass magnetisation in zero-field magnetisation experiments, $M_{\mathrm{ZFC}}\left(t, t_{\mathrm{w}} ; T\right)$, is measured from simulations, verifying the scaling relationships in the dynamical or non-equilibrium regime. Our preliminary search for the de Almeida-Thouless line in $D=3$ is discussed.

Keywords: ergodicity breaking, memory effects, spin glasses

\section{Contents}

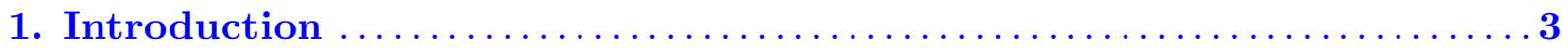

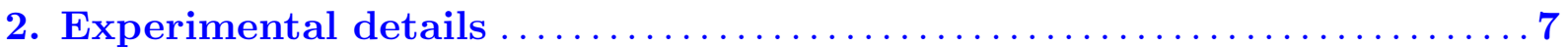




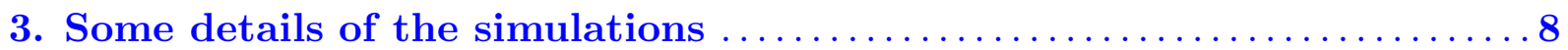

4. Measurements and computations of the relaxation rate $\ldots \ldots \ldots \ldots \ldots 9$

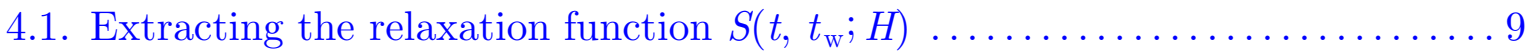

4.2. A different approach for the computation of $t_{H}^{\mathrm{eff}}$ in simulations ........... 11

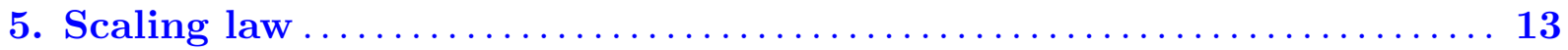

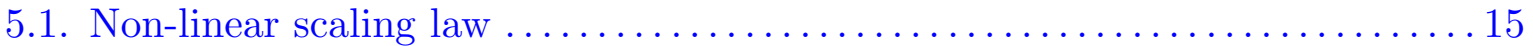

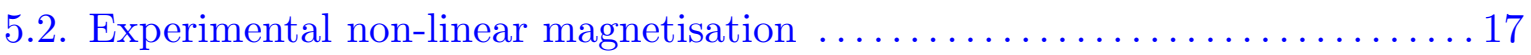

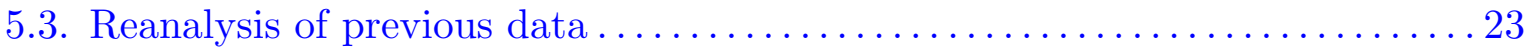

5.4. Numerical study of the ratio of the effective times at $\mathrm{H}$ and $\mathrm{H}=0^{+} \ldots \ldots 25$

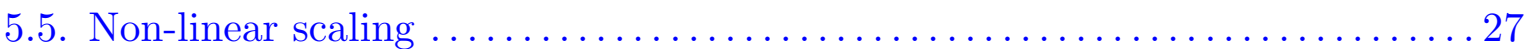

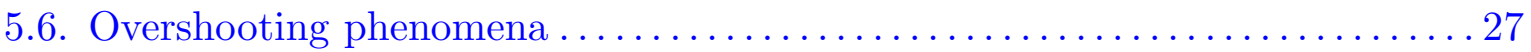

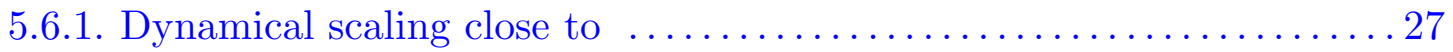

5.6.2. Overshooting in a ferromagnetic system .................... 29

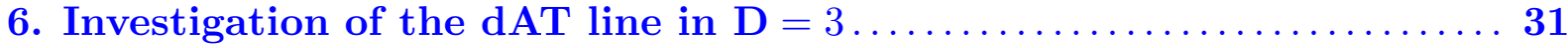

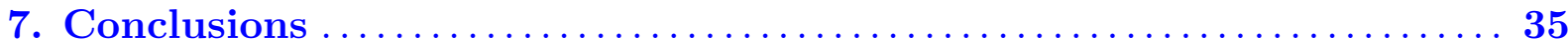

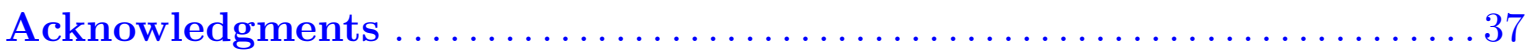

Appendix A. Technical details about our simulations 38

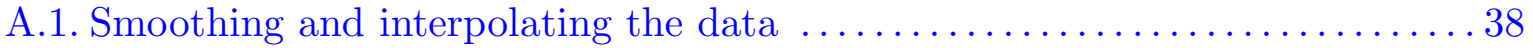

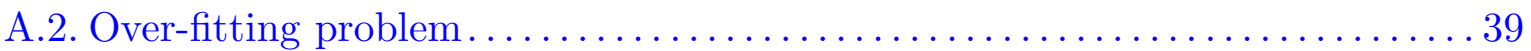

A.3. Time discretisation and the calculation of the relaxation function

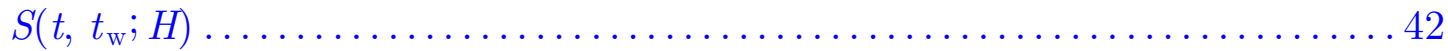

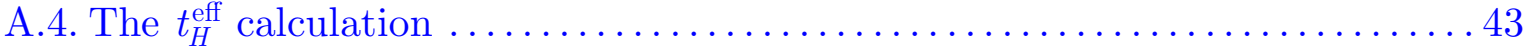

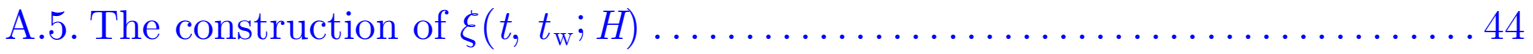

Appendix B. The Josephson length 44

Appendix C. Sample dependence of the non-linear scaling results 45

$\begin{array}{ll}\text { References } & 47\end{array}$

\section{Introduction}

This paper examines in detail the dynamics of spin glasses in the vicinity of and below their condensation temperature $T_{\mathrm{g}}$ in the presence of a magnetic field, an exploration relevant to many other condensed-matter glassy systems: fragile molecular glasses, polymers, colloids, super-cooled liquids, and now even social science through 'resource dynamics on species packing in diverse ecosystems' [1], to name a few. The advent of realistic time and length scales on the Janus II dedicated supercomputer generates a synergy between theory, experiment, and simulations that encompasses a thorough 
examination of spin-glass dynamics reaching from the low-temperature regime to the vicinity of $T_{\mathrm{g}}[2-4]$.

The special nature of this approach is the use of the spin-glass correlation length, $\xi\left(t, t_{\mathrm{w}} ; T\right)$ as the primary factor in the analysis [4], where $t$ is the time after a magnetic field change, when measurements of the magnetisation take place; $t_{\mathrm{w}}$ is the waiting time after the system is quenched from above $T_{\mathrm{g}}$ to temperatures within the condensation regime, and before the change in magnetic field; and $T$ is the temperature. This correlation length can be extracted from experiment and simulations, under dynamical or non-equilibrium configurations [5-8]. As such, it will be used for a detailed study of a new powerful scaling law for the non-linear magnetisation near to and below $T_{\mathrm{g}}$. We shall show that this law not only accounts for the experiments presented in this paper, but also clears up some historical issues about the nature of the Zeeman, or magnetic-field, energy in the spin-glass state [5, 9].

To be specific about the temperature and magnetic field protocol, we present an analysis of the zero-field-cooled magnetisation, $M_{\mathrm{ZFC}}\left(t, t_{\mathrm{w}} ; H\right)$, as a function of $t, t_{\mathrm{w}}$ and magnetic field $H$ at prescribed temperatures $T \leqslant T_{\mathrm{g}}$. The protocol is one where the 'sample' is quenched from a temperature $T>T_{\mathrm{g}}$ to a measuring temperature $T \leqslant T_{\mathrm{g}}$ in zero magnetic field. The word quench means different things experimentally and in simulations. In the former, there is a finite cooling rate as the system is brought from above $T_{\mathrm{g}}$ to the measurement temperature $T$. It is typically of the order of one to tens of seconds per degree of cooling. In the case of simulations, it is instantaneous. Though on the surface this would seem a difficult issue, in fact temperature chaos $[10,11]$ (which we now know to be present in non-equilibrium dynamics as well [12]) makes the two approaches similar if not identical. Experimentally, though the cooling rate is finite, lowering the temperature sufficiently ( $\delta T$ larger than milli-Kelvins) creates new spinglass states without knowledge of previous history (a process termed rejuvenation [13]). This is the reason that the magnetic susceptibility is reproducible from one experiment to another, without recourse to the cooling rate. Thus, the final state reached upon an experimental temperature quench is as fresh as the state arrived at in simulations upon instantaneous quenching.

After the measurement temperature $T$ is reached, the system is held for a time $t_{\mathrm{w}}$, the waiting time, after which a magnetic field $H$ is turned on. The resulting magnetisation, $M_{\mathrm{ZFC}}\left(t, t_{\mathrm{w}} ; H\right)$, is then measured over a time interval $t$. The response consists of two terms: an instantaneous increase in magnetisation (the so-called reversible magnetisation), and a slowly increasing part termed the irreversible magnetisation. The latter is found to depend upon all of the factors $t, t_{\mathrm{w}}, H$. The rise of the irreversible term is typically very slow, taking literally times of the order of the age of the Universe to reach equilibrium. For this reason, a spin glass, once perturbed from a quasiequilibrium state, never reaches equilibrium, and an experiment is always in a dynamical or non-equilibrium regime.

The sum of the reversible and irreversible magnetisations grows towards a welldefined 'target', the so-called field-cooled magnetisation, $M_{\mathrm{FC}}$, for which the measuring protocol is the opposite of the zero-field magnetisation. Namely, at $T>T_{\mathrm{g}}$, a magnetic field $H$ is turned on, and then the temperature is quenched to $T \leqslant T_{\mathrm{g}}$. Typically, $M_{\mathrm{FC}}$ is relatively constant, but not without its own dynamics. If the magnetic field is suddenly 
removed, the magnetisation immediately decays by its reversible part (the same as in the zero-field case), followed by a slow decay termed the irreversible part or $M_{\mathrm{TRM}}\left(t, t_{\mathrm{w}} ; H\right)$, the thermo-remanent magnetisation dependent upon the waiting time $t_{\mathrm{w}}$. In general, it is found that

$$
M_{\mathrm{FC}}=M_{\mathrm{ZFC}}\left(t, t_{\mathrm{w}} ; H\right)+M_{\mathrm{TRM}}\left(t, t_{\mathrm{w}} ; H\right) .
$$

This is known as the extended principle of superposition [14]. There is an immense literature covering both $M_{\mathrm{ZFC}}\left(t, t_{\mathrm{w}} ; H\right)$ and $M_{\mathrm{TRM}}\left(t, t_{\mathrm{w}} ; H\right)$ measurements, and the physical insights gained from them [14-16].

Our approach is rather different, in that we choose to represent the dynamics in terms of the spin-glass correlation length $\xi\left(t, t_{\mathrm{w}} ; H\right)$. This quantity was first extracted from experiment in the work of Joh et al [5], who developed a protocol based on the relaxation function $S\left(t, t_{\mathrm{w}} ; H\right)$ defined by

$$
S\left(t, t_{\mathrm{w}} ; H\right)=\mathrm{d}\left[\frac{-M_{\mathrm{TRM}}\left(t, t_{\mathrm{w}} ; H\right)}{H}\right] / \mathrm{d} \ln t .
$$

It is known that $S\left(t, t_{\mathrm{w}} ; H\right)$ peaks at what is termed an effective waiting time, $t_{H}^{\mathrm{eff}}$, which is usually of the order of $t_{\mathrm{w}}$ [17]. This time is characteristic of the decay of $M_{\mathrm{TRM}}\left(t, t_{\mathrm{w}} ; H\right)$, or, through equation (1), of the increase of $M_{\mathrm{ZFC}}\left(t, t_{\mathrm{w}} ; H\right)$ with time $t$. As noted by Lederman et al [18] and Hammann et al [19] for states distributed according to ultrametric symmetry, the dynamics is controlled by a largest free-energy barrier height, $\Delta_{\max }$, associated with the state that has the smallest overlap with the initial state, $q_{\min }$. Thus, $t_{H}^{\text {eff }}$ can be associated with $\Delta_{\max }$ through the usual Arrhenius law:

$$
\Delta_{\max }=k_{\mathrm{B}} T\left(\ln t_{H}^{\mathrm{eff}}-\ln \tau_{0}\right),
$$

where $\tau_{0}$ is a characteristic exchange time, $\tau_{0} \sim \hbar / k_{\mathrm{B}} T_{\mathrm{g}}$.

In order to extract a correlation length, Joh et al [5] used the notion that the freeenergy barrier heights were reduced in the presence of a magnetic field by the Zeeman energy, $E_{\mathrm{Z}}[5,20-23]$, and that, for small magnetic fields $H$,

$$
E_{\mathrm{Z}}=\left(V_{\text {corr }} / a_{0}^{3}\right) \chi_{\mathrm{FC}} H^{2}
$$

where $\chi_{\mathrm{FC}}$ is the field-cooled magnetic susceptibility per spin, $V_{\text {corr }}$ is the correlated volume, and $a_{0}$ the average spatial separation of the magnetic ions, so that the number of correlated spins is $N_{\text {corr }}=V_{\text {corr }} / a_{0}^{3}$. Joh et al took

$$
N_{\text {corr }}=V_{\text {corr }} / a_{0}^{3} \approx \frac{4 \pi}{3} \xi^{3},
$$

where $\xi$ is in units of $a_{0}$. We now know that a more appropriate relationship would be

$$
N_{\text {corr }}=V_{\text {corr }} / a_{0}^{3}=\frac{4 \pi}{3} \xi^{3-\theta(\tilde{x}) / 2} \equiv b \xi^{3-\theta(\tilde{x}) / 2},
$$

where $b$ is a geometrical factor, and $\theta(\tilde{x})$ is the replicon exponent [23]. Using equation (2) through (4), the experiments in [5] produced the plot in figure 1. The data in the limit of small magnetic fields $H$ were clearly linear in $H^{2}$, allowing equation (4) through (6) to set a value for $\xi$. 
Spin-glass dynamics in the presence of a magnetic field: exploration of microscopic properties

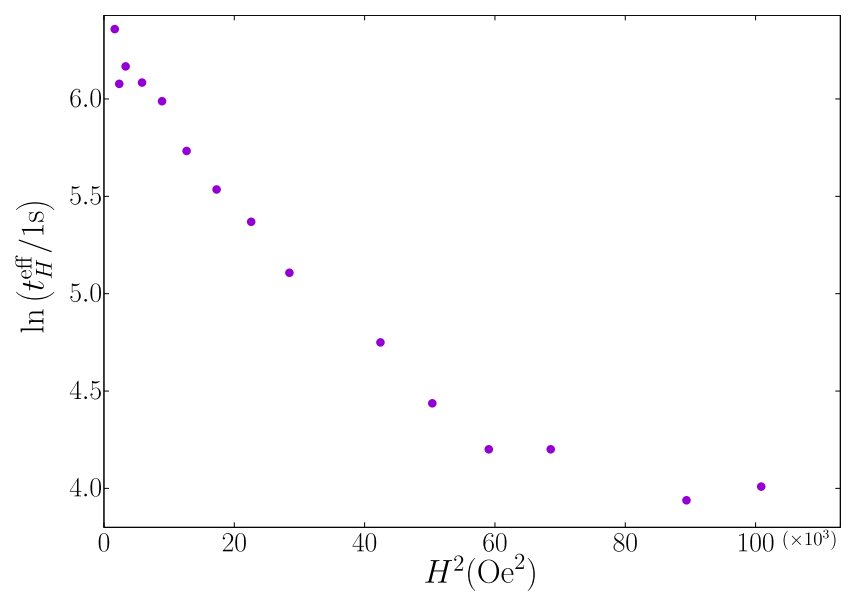

Figure 1. A plot of $\ln t_{H}^{\mathrm{eff}}$, extracted from the Zeeman energy $E_{\mathrm{Z}}$ as in equation (4), vs $H^{2}$ for Cu:Mn 6 at. $\%\left(T / T_{\mathrm{g}}=0.83, t_{\mathrm{w}}=480 \mathrm{~s}\right)$ at fixed $t_{\mathrm{w}}$ and $T$. Data taken from [5].

The deviation from linearity in $H^{2}$ was puzzling, leading to the authors' stating: 'we do not have a satisfactory explanation for this change in slope. A different description predicts a linear dependence of $E_{\mathrm{Z}}$ upon $H$, which can be made to fit the data $(.$. but with a significant deviation at small field changes'. It is the purpose of this paper to analyse the entirety of the data considering non-linear terms in the spin-glass magnetisation according to a new scaling law. In addition to analysing magnetisation data from new experiments, we shall also show that the data of figure 1, and subsequent experiments of Bert et al [9] on the Ising spin glass $\mathrm{Fe}_{0.5} \mathrm{Mn}_{0.5} \mathrm{TiO}_{3}$, fit the new scaling law well, obviating the need to question equation (4), and putting to rest the controversy over the nature of the Zeeman energy in spin glasses.

This paper brings together a complete set of magnetisation measurements of a single crystal of the prototypical spin glass, CuMn 6 at. \%. The beauty of the experimental results is that the correlated volume is most certainly spherical (as opposed to thin films where the correlated volume is of pancake geometry [6]), and unlimited by finite-size crystallites separated by grain boundaries [24, 25]. Accompanying these measurements are the remarkable simulations of the Janus II dedicated supercomputer, which, for the first time, yield spin-glass correlations that approach experimental time and length scales. Indeed, the range of correlation lengths that we are able to achieve both experimentally and numerically is itself a breakthrough. The current manuscript reports results up to $\xi \approx 23.6 a_{0}$ ( $a_{0}$ is the typical $\mathrm{Mn}-\mathrm{Mn}$ distance), which represents a step forward by a factor of three from previous work [23]. On the experimental side, we reach a correlation length four times larger than in [5].

The synergy between these two approaches, combined with theory, opens up a new vista for spin-glass dynamics. A direct outgrowth of this collaboration is the introduction of the new magnetisation scaling law, which encompasses the full range of magnetic fields for temperatures in the vicinity of the condensation temperature $T_{\mathrm{g}}$ [4]. This scaling law successfully describes both experimental and simulation results and, as noted above, 
Spin-glass dynamics in the presence of a magnetic field: exploration of microscopic properties

Table 1. The values of the measuring temperatures $T_{\mathrm{m}}$ and waiting times $t_{\mathrm{w}}$ for the four experimental regimes, the respective correlation lengths at times $t_{\mathrm{w}}$ (in units of the average $\mathrm{Mn}-\mathrm{Mn}$ spacing $\left.a_{0}\right)$, and the effective replicon exponent $\theta(\tilde{x})$ obtained from equations (41) and (42) below (see also appendix B).

\begin{tabular}{lcccc}
\hline & $T_{\mathrm{m}}(\mathrm{K})$ & $t_{\mathrm{w}}(\mathrm{s})$ & $\xi\left(t_{\mathrm{w}}\right) / a_{0}$ & $\theta(\tilde{x})$ \\
\hline Exp. 1 & 28.50 & 10000 & 320.36 & 0.337 \\
Exp. 2 & 28.75 & 10000 & 341.76 & 0.344 \\
Exp. 3 & 28.75 & 20000 & 359.18 & 0.342 \\
Exp. 4 & 29.00 & 10000 & 391.27 & 0.349 \\
\hline
\end{tabular}

will resolve a nearly three-decade-old controversy concerning the nature of the magnetic state.

Although our simulations were not designed to that end, we take the occasion as well to attempt a preliminary search for the de Almeida-Thouless (dAT) line in the phase diagram of the 3D spin glass.

The paper is organised as follows. Section 2 details the experimental measurements of the non-linear magnetisation in the CuMn spin glass. Section 3 describes the nature of our numerical simulations. Section 4 introduces the response function, equation (2), and its extraction from experiment and simulations. Section 5 develops the new scaling law, and applies it to both experimental and simulation results. In addition, section 5.6 shows the nature of the growth of the numerical correlation length $\xi$ in the presence of a magnetic field at temperatures close to the critical temperature $T_{\mathrm{g}}$. We observed interesting overshoot phenomena that we prove to be general, as they are observed even in ferromagnetic systems. Section 6 investigates the dAT phase boundary in $D=3$. Important technical details are provided in the appendices. Finally, section 7 summarises our results, and points to future opportunities stemming from the synergy expressed in this paper between theory, experiment, and simulations.

\section{Experimental details}

The experimental measurements were made with a $\mathrm{CuMn} \sim 6$ at. $\%$ single-crystal sample, prepared using a Bridgman method. The $\mathrm{Cu}$ and $\mathrm{Mn}$ were arc melted several times in an argon environment and cast in a copper mould. The ingot was then processed in a Bridgman furnace. Both x-ray fluorescence and optical observation showed that the beginning of the growth is a single phase. More details can be found in appendix A of [3]. The transition temperature, $T_{\mathrm{g}}=31.5 \mathrm{~K}$, was determined from the temperature at which $M_{\mathrm{ZFC}}(T)$ first began to depart from $M_{\mathrm{FC}}(T)$.

The magnetisation measurements were made using a commercial DC SQUID. The sample was quenched from $40 \mathrm{~K}$ at $10 \mathrm{~K} \mathrm{~min}^{-1}$ to the measuring temperature $T_{\mathrm{m}}$ in zero magnetic field. After stabilisation of the temperature, the system was aged for a waiting time $t_{\mathrm{w}}$ before a magnetic field was applied, and the magnetisation $M_{\mathrm{ZFC}}\left(t, t_{\mathrm{w}} ; T_{\mathrm{m}}\right)$ recorded as a function of time $t$. The temperatures $T_{\mathrm{m}}$ were chosen as $28.5 \mathrm{~K}, 28.75 \mathrm{~K}$, and $29 \mathrm{~K}$, so that $T_{\mathrm{m}} \geqslant 0.9 T_{\mathrm{g}}$. The magnetic fields ranged from 16 Oe to 59 Oe. 
Spin-glass dynamics in the presence of a magnetic field: exploration of microscopic properties

Table 2. Main parameters for each of our numerical simulations: $T, t_{\mathrm{w}}, \xi\left(t_{\mathrm{w}}\right)$, the longest simulation time $t_{\max }$, the replicon exponent $\theta(\tilde{x})$ (see appendix $\mathrm{B}$ ) and the value of $C_{\text {peak }}\left(t_{\mathrm{w}}\right)$ employed in equation (16). Here and in the rest of the paper, error bars are one standard deviation.

\begin{tabular}{lcccccc}
\hline & $T$ & $t_{\mathrm{w}}$ & $\xi\left(t_{\mathrm{w}} ; H=0\right)$ & $t_{\max }$ & $\theta(\tilde{x})$ & $C_{\text {peak }}$ \\
\hline Run 1 & 0.9 & $2^{22}$ & $8.294(7)$ & $2^{30}$ & 0.455 & $0.533(3)$ \\
Run 2 & 0.9 & $2^{26.5}$ & $11.72(2)$ & $2^{30.5}$ & 0.436 & $0.515(2)$ \\
Run 3 & 0.9 & $2^{31.25}$ & $16.63(5)$ & $2^{33}$ & 0.415 & $0.493(3)$ \\
Run 4 & 1.0 & $2^{23.75}$ & $11.79(2)$ & $2^{28}$ & 0.512 & $0.422(2)$ \\
Run 5 & 1.0 & $2^{27.625}$ & $16.56(5)$ & $2^{30}$ & 0.498 & $0.400(1)$ \\
Run 6 & 1.0 & $2^{31.75}$ & $23.63(14)$ & $2^{35}$ & 0.484 & $0.386(4)$ \\
\hline
\end{tabular}

Table 1 displays the relevant experimental parameters, including the effective replicon exponent $\theta(\tilde{x})$.

\section{Some details of the simulations}

We carried out massive simulations on the Janus II supercomputer [26] to study the Ising-Edwards-Anderson (IEA) model in a cubic lattice with periodic boundary conditions and size $L=160 a_{0}$, where $a_{0}$ is the average distance between magnetic moments, see table 2 for the simulation details. The $N=L^{D}$ Ising spins, $s_{x}= \pm 1$, interact with their lattice nearest neighbours through the Hamiltonian:

$$
\mathcal{H}=-\sum_{\langle\boldsymbol{x}, \boldsymbol{y}\rangle} J_{x y} s_{x} s_{x}-H \sum_{x} s_{x}
$$

where the quenched disordered couplings are $J_{x y}= \pm 1$ with $50 \%$ probability. We name a particular choice of the couplings a sample. In the absence of an external magnetic field, $H=0$, this model undergoes a spin-glass transition at the critical temperature $T_{\mathrm{g}}=1.102(3)[27]$.

As we explained in the introduction, in order to simulate the experimental zerofield-cooling (ZFC) protocol the following procedure was performed: we place the initial random spin configuration instantaneously at the working temperature $T$ and let it relax for a time $t_{\mathrm{w}}$ at $H=0$. At time $t_{\mathrm{w}}$, we turn on the external magnetic field and we start recording the magnetic density,

$$
M_{\mathrm{ZFC}}\left(t, t_{\mathrm{w}} ; H\right)=\frac{1}{160^{3}} \sum_{x} s_{x}\left(t+t_{\mathrm{w}} ; H\right),
$$

as well as the correlation function,

$$
C\left(t, t_{\mathrm{w}} ; H\right)=\frac{1}{160^{3}} \sum_{x} s_{x}\left(t_{\mathrm{w}} ; 0\right) s_{x}\left(t+t_{\mathrm{w}} ; H\right) .
$$


The non-equilibrium dynamics was simulated with a Metropolis algorithm; the numerical time unit being the lattice sweep, roughly corresponding to 1 ps of physical time [28]. For each temperature and waiting time, see table 2, several magnetic fields were simulated. For computational reasons, one single independent sample was simulated for each case. We checked, however, the robustness and the sample independence of our results in a single case, studied in detail in appendix $\mathrm{C}$.

According to reference [23], the value of the dimensionless magnetic field $H$ used in the numerical simulation can be matched to the physical field. This relation was estimated from experimental $\mathrm{Fe}_{0.5} \mathrm{Mn}_{0.5} \mathrm{TiO}_{3}$ data [29]. We found that $H=1$ in the IEA model corresponded to $\approx 5 \times 10^{4}$ Oe physically. Hence, our experimental range $(16$ Oe to 59 Oe) corresponds to magnetic field $0.0003 \lesssim H \lesssim 0.0012$ in the IEA model. However, the signal-to-noise ratio, which scales linearly in $H$ for small fields [30], limited our simulation to $H \geqslant 0.005$, equivalent to a physical $H=250 \mathrm{G}$.

In order to match the experimental and numerical scales, we exploited dimensional analysis [31] to relate $H$ and the reduced temperature $\hat{t}=\left(T_{\mathrm{g}}-T\right) / T_{\mathrm{g}}$ through the scaling

$$
\hat{t}_{\text {num }} \approx \hat{t}_{\exp }\left(\frac{H_{\text {num }}}{H_{\exp }}\right)^{\frac{4}{\nu(5-\eta)}},
$$

where $\nu=2.56(4)$ and $\eta=-0.39(4)$ are $H=0$ critical exponents [27], while subscripts exp and num stand for experiment and simulation, respectively. According to equation (10), and minding signal-to-noise limitations, we can match the experimental and numerical scales by increasing $\hat{t}_{\text {num }}$, resulting in $0.89 \lesssim T_{\text {num }} \lesssim 0.99$. Given our pre-existing database of long simulations at $H=0$ [2], it has been convenient to work at temperatures $T_{\text {num }}=0.9$ and $T_{\text {num }}=1.0$. Table 2 displays the relevant numerical parameters, including the effective replicon exponent $\theta(\tilde{x})$ and the $C_{\text {peak }}\left(t_{\mathrm{w}}\right)$ values that will be introduced and explained in section 4.2.

Let us finally remark that section 5.6 uses a different set of simulations from the rest of the paper.

\section{Measurements and computations of the relaxation rate}

In this section we describe the relaxation function $S\left(t, t_{\mathrm{w}} ; H\right)$ (section 4.1) and explain how $t_{H}^{\text {eff }}$ is extracted from simulations (section 4.2).

\subsection{Extracting the relaxation function $S\left(t, t_{\mathrm{w}} ; H\right)$}

The main quantity used in our experiments of [5] is the relaxation function $S\left(t, t_{\mathrm{w}} ; H\right)$ :

$$
S\left(t, t_{\mathrm{w}} ; H\right)=\frac{\mathrm{d} M_{\mathrm{ZFC}}\left(t, t_{\mathrm{w}} ; H\right)}{\mathrm{d} \ln t},
$$

which exhibits a local maximum at time $t_{H}^{\text {eff }}$. Experimentally, measurements of $M_{\mathrm{ZFC}}\left(t, t_{\mathrm{w}} ; H\right)$ enable the evaluation of the relaxation function $S\left(t, t_{\mathrm{w}} ; H\right)$ directly. A 
Spin-glass dynamics in the presence of a magnetic field: exploration of microscopic properties

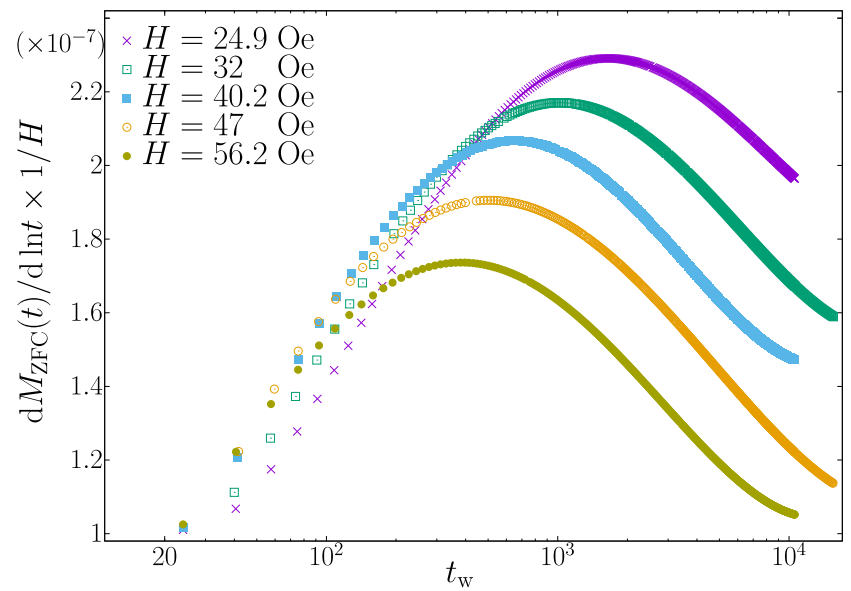

Figure 2. Example of $S\left(t, t_{\mathrm{w}} ; H\right)$ measurements for different magnetic fields. The sample is a single crystal CuMn 6 at. \%, and the measurements were taken at a waiting time of $t_{\mathrm{w}}=10000 \mathrm{~s}$ and at $T=28.5 \mathrm{~K}$. The time at which $S\left(t, t_{\mathrm{w}} ; H\right)$ peaks defines $t_{H}^{\text {eff }}$, the effective response time. The shift to shorter times as $H$ increases is the measure of the reduction of $\Delta_{\max }$ with increasing Zeeman interaction, and is used to extract the linear and non-linear terms in the magnetic susceptibility.

representative set of data for $T_{\mathrm{m}}=28.5 \mathrm{~K}$ and $t_{\mathrm{w}}=10^{4} \mathrm{~s}$ is displayed in figure 2 . Numerically, the calculation of $S\left(t, t_{\mathrm{w}} ; H\right)$ is sensitive to the relative errors of the magnetisation density, which increase as

$$
\frac{\delta M_{\mathrm{ZFC}}\left(t, t_{\mathrm{w}} ; H\right)}{M_{\mathrm{ZFC}}} \propto \frac{1}{H} .
$$

We employ two tricks to extract the relaxation function $S\left(t, t_{\mathrm{w}} ; H\right)$ from simulations. On the one hand, we perform a de-noising method to regularise the magnetisation density $M_{\mathrm{ZFC}}\left(t, t_{\mathrm{w}} ; H\right)$, exploiting the fluctuation-dissipation relations (FDRs) [32-36]

$$
\frac{T}{H} M_{\mathrm{ZFC}}\left(t, t_{\mathrm{w}} ; H\right)=\mathcal{F}(C ; H),
$$

where $\mathcal{F}(C ; H)$ behaves at large $C\left(t, t_{\mathrm{w}} ; H\right)$ as $\mathcal{F}(C ; H)=1-C\left(t, t_{\mathrm{w}} ; H\right)$. We report the details in appendix A.1. On the other hand, we define $S\left(t, t_{\mathrm{w}} ; H\right)$ as a finite-time difference

$$
S\left(t, t_{\mathrm{w}}, t^{\prime} ; H\right)=\frac{M_{\mathrm{ZFC}}\left(t^{\prime}, t_{\mathrm{w}} ; H\right)-M_{\mathrm{ZFC}}\left(t, t_{\mathrm{w}} ; H\right)}{\ln \left(t^{\prime} / t\right)} .
$$

In simulations, time is discrete and we store configurations at times $t_{n}=$ integer-part-of $2^{n / 4}$, with $n$ an integer. Let us write explicitly the integer dependence of times $t$ and $t^{\prime}$ as:

$$
t \equiv t_{n}, \quad t^{\prime} \equiv t_{n+k}
$$

where $k$ is an integer time parameter. The reader will note that there is a trade-off in the choice of $k$. On the one hand, the smaller $k$ is the better the finite difference in 
Spin-glass dynamics in the presence of a magnetic field: exploration of microscopic properties

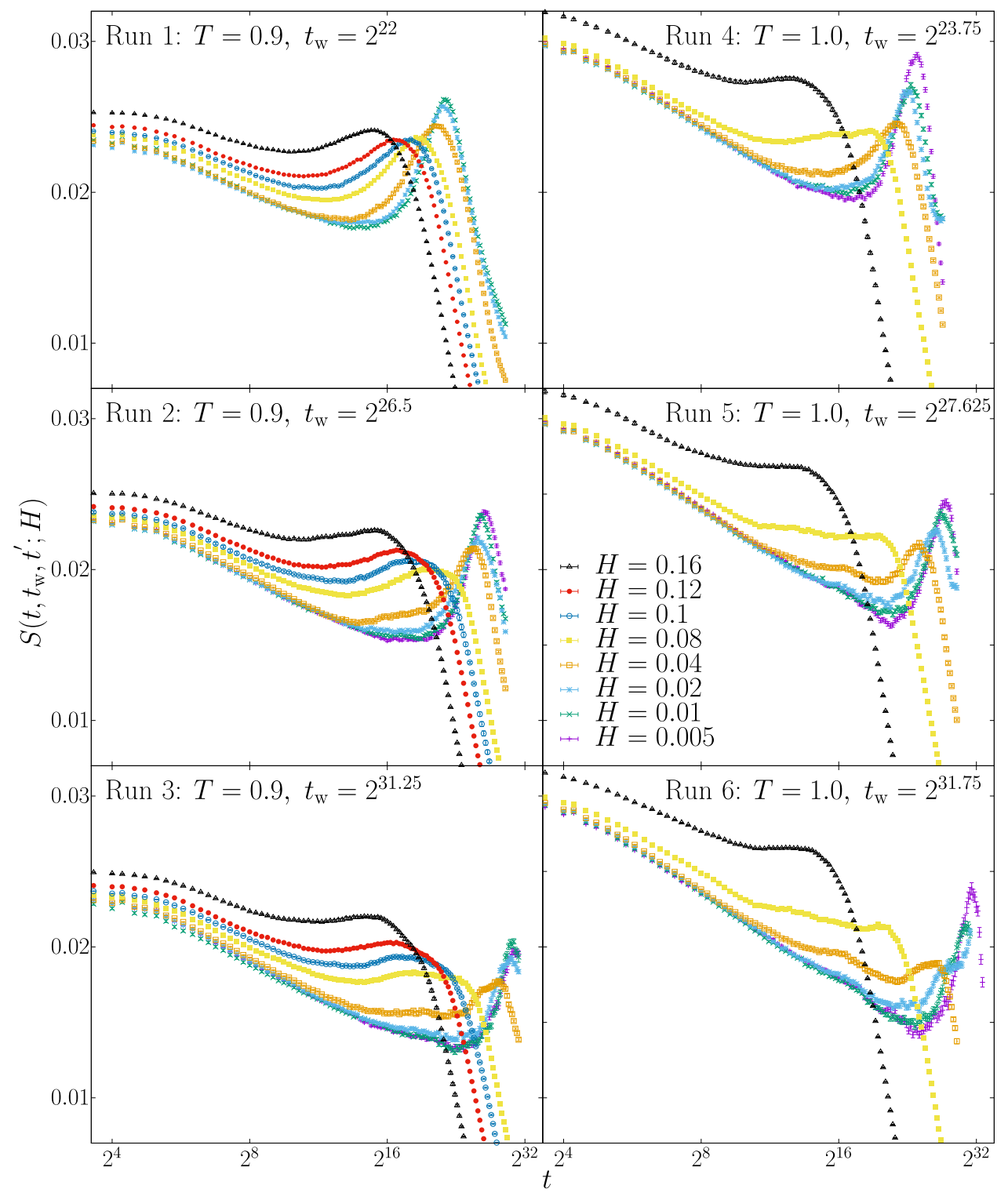

Figure 3. Time evolution of the relaxation rate $S\left(t, t_{\mathrm{w}}, t^{\prime} ; H\right)$ of equation (14) for the six runs of table 2. All plots have the time parameter $k=8$ in equation (15).

equation (14) represents the derivative. On the other hand, when $k$ grows the statistical error in the evaluation of equation (14) decreases significantly. In this section we report only the case for $k=8$ (more details about time discretisation are provided in appendix A.3). The numerical $S\left(t, t_{\mathrm{w}}, t^{\prime} ; H\right)$ are exhibited in figure 3 , where a local maximum in the long-time region can be seen.

\subsection{A different approach for the computation of $t_{H}^{\text {eff }}$ in simulations}

As explained in the introduction, we are interested in the evaluation of the time when the relaxation function $S\left(t, t_{\mathrm{w}}, t^{\prime} ; H\right)$ peaks, namely $t_{H}^{\mathrm{eff}}$. Two problems arise: 
(a) The reader will note two separate peaks in the $S\left(t, t_{\mathrm{w}}, t^{\prime} ; H\right)$ curves of figure 3 : namely the peak at microscopic times $t \sim 4$, and the peak we are interested in at $t \sim t_{\mathrm{w}}$. Unfortunately, the distinction between the two is only clear at small $H$. Previous numerical work [23] did not face this problem, probably because of their smaller correlation length, $\xi \approx 8$ (non-linear susceptibilities grow very fast with $\xi$, see the next section).

(b) We are most interested in the limit $H \rightarrow 0$, which is extremely noisy, as we have explained above.

An interesting possibility emerges when plotting the relaxation function $S\left(t, t_{\mathrm{w}}, t^{\prime} ; H\right)$ in terms of the correlation function $C\left(t, t_{\mathrm{w}} ; H\right)$, rather than as a function of time (see figure 4$). C\left(t, t_{\mathrm{w}} ; H\right)$ is a decreasing function of time, the long-time region corresponding to small $C\left(t, t_{\mathrm{w}} ; H\right)$, and vice versa. Hence, the physical peak in which we are interested is the one that appears at small $C\left(t, t_{\mathrm{w}} ; H\right)$ (see figure 4$)$. Analogously to figure 3, we report only the case for $k=8$ in figure 4 .

The simulation data strongly suggest that, when $H \rightarrow 0$, the correlation function $C\left(t, t_{\mathrm{w}} ; H\right)$ approaches a constant value $C_{\text {peak }}\left(t_{\mathrm{w}}\right)$ at the maximum of the relaxation function. Hence, our proposal is to define $t_{H}^{\mathrm{eff}}$ in simulations as the time when $C\left(t, t_{\mathrm{w}} ; H\right)$ reaches the value $C_{\text {peak }}\left(t_{\mathrm{w}}\right)$ :

$$
C\left(t^{\mathrm{eff}}{ }_{H}, t_{\mathrm{w}} ; H\right)=C_{\text {peak }}\left(t_{\mathrm{w}}\right) .
$$

As the reader can see, equation (16) is applicable also at $H=0$, solving the problem of the vanishing magnetisation in this limit. The crucial point for our new $t_{H}^{\mathrm{eff}}$ definition, see equation (16), is, hence, the computation of $C_{\text {peak }}\left(t_{\mathrm{w}}\right)$. Two problems arise:

(a) The constant-value $C_{\text {peak }}\left(t_{\mathrm{w}}\right)$ is well defined only for small magnetic field $H$.

(b) The relaxation function as a function of the correlation, $\mathcal{S}(C ; H)$, is an implicit function of a reparametrised time,

$$
t_{\text {new }}=\frac{1}{2} \ln \left(\frac{t_{n+k}}{t_{n}}\right)
$$

(see appendix A.3 for details).

Our strategy has been to study, for each run, the behaviour of $\mathcal{S}(C ; H)$ for the two smallest magnetic fields $H$ and for three different time parameters $k$. We report in figure 5 a closeup of the peak region for $\mathcal{S}(C ; H)$, used for the evaluation of $C_{\text {peak }}\left(t_{\mathrm{w}}\right)$. We report our estimates for $C_{\text {peak }}\left(t_{\mathrm{w}}\right)$ in table 2 .

The relaxation function $S\left(t_{\text {new }}, t_{\mathrm{w}} ; H\right)$ depends on the correlation length $\xi\left(t_{\mathrm{w}}\right)$, and on the applied magnetic field $H$, equation (2). We observe, however, that $S\left(t_{\text {new }}, t_{\mathrm{w}} ; H\right)$ has a temperature dependence, which we extract by comparing runs 4 and 2 in figure 5 . These two cases are characterised by

(a) A similar starting correlation length $\xi\left(t_{\mathrm{w}} ; H=0\right) \approx 11.7$ (see table 2 ),

(b) The same set of applied magnetic fields, namely $H=0.005$ and $H=0.01$. 
Spin-glass dynamics in the presence of a magnetic field: exploration of microscopic properties

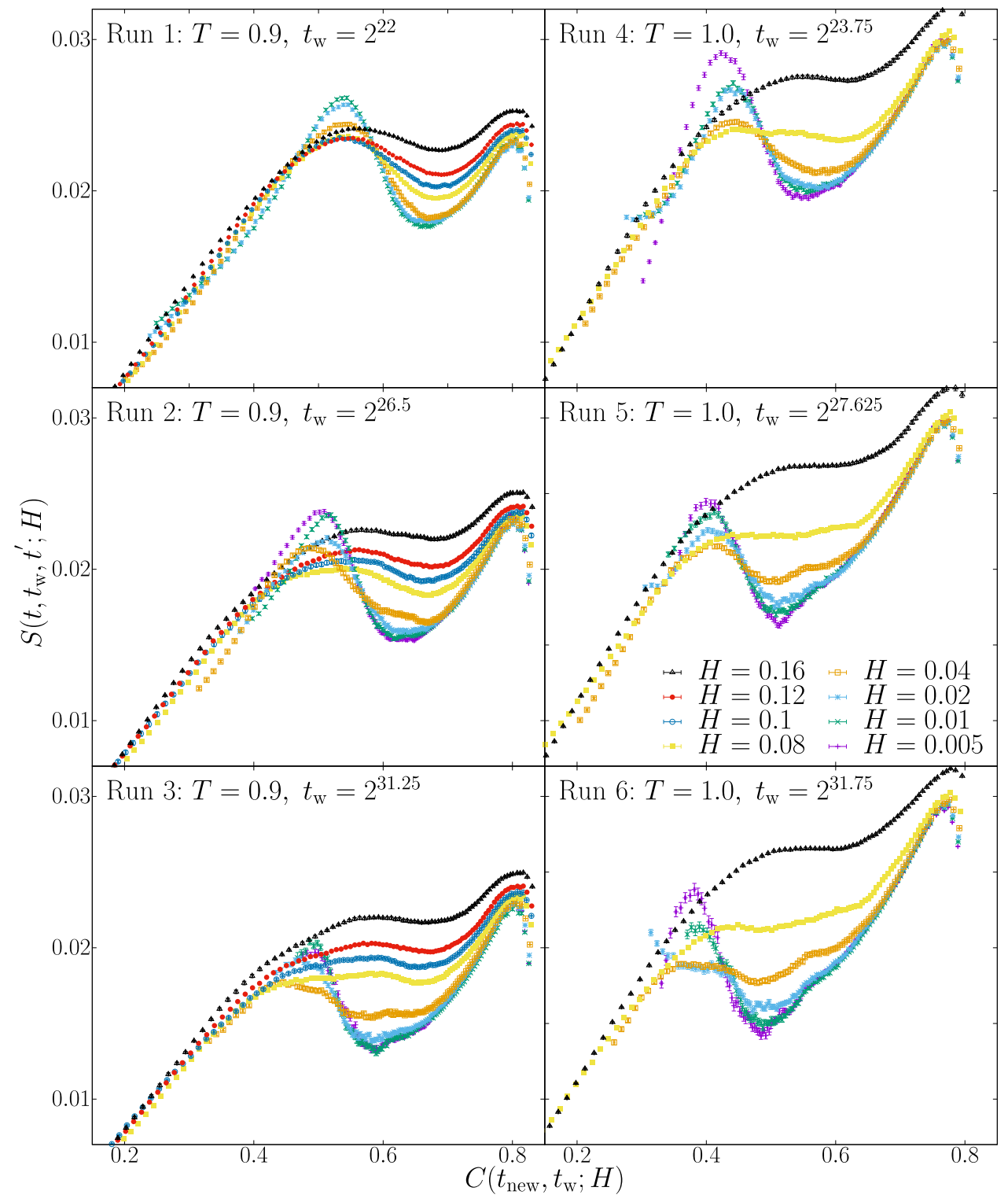

Figure 4. $S\left(t, t_{\mathrm{w}}, t^{\prime} ; H\right)$ as a function of $C\left(t, t_{\mathrm{w}} ; H\right)$. The peak region is enlarged in figure 5 below. The physically relevant peak is the one for small $C$, corresponding to long times. We consider the reparametrised $t_{\text {new }}$ with $k=8$ in equation (17).

Yet, there appear to be two different scenarios in the data plotted in figure 5 . In run 2 , the peak of $\mathcal{S}(C ; H)$ is almost the same for all the rescaled time curves. In run 4 , however, the peaks separate for different $k$. As will be explained in section 5.5, this difference in behaviour is caused by increasing non-linear effects in the magnetisation, $M_{\mathrm{ZFC}}\left(t, t_{\mathrm{w}} ; H\right)$.

In conclusion, equation (16) solves our two problems at once. We no longer need to resolve the short-time and the long-time peaks in figure 3 and it bypasses the problem of the vanishing magnetisation as $H$ goes to zero. 
Spin-glass dynamics in the presence of a magnetic field: exploration of microscopic properties

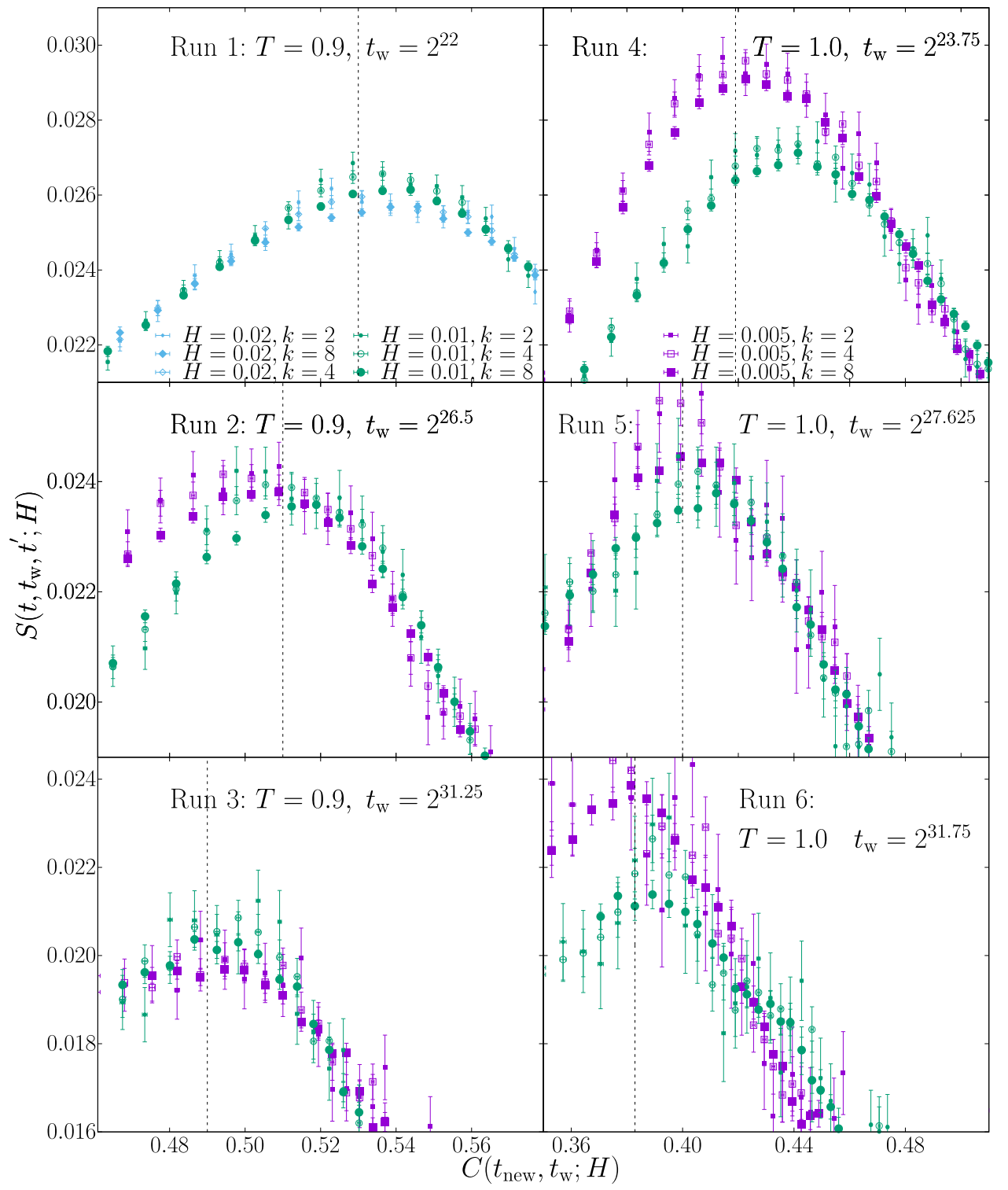

Figure 5. Enlargement of the peak region of $S\left(t, t_{\mathrm{w}}, t^{\prime} ; H\right)$ as a function of $C\left(t, t_{\mathrm{w}} ; H\right)$ for several values of time parameter $k$ in equation (15). The dashed black lines indicate the $C_{\text {peak }}\left(t_{\mathrm{w}}\right)$ positions.

\section{Scaling law}

We address here three different aspects of the scaling law. The assumptions that led us to our scaling law are given in section 5.1. Next, in section 5.2, we use the scaling law in the analysis of our experimental data (previous data are also reanalysed in section 5.3), with the corresponding analysis for our simulations given in section 5.4. Section 5.5 shows our experimental and numerical results together, according to the new scaling law. In section 5.6 we address the nature of the growth of the numerical correlation length 
$\xi\left(t_{\mathrm{w}}\right)$ in the presence of a magnetic field at temperatures close to the condensation temperature $T_{\mathrm{g}}$.

\subsection{Non-linear scaling law}

Scaling laws for the spin-glass susceptibility in the vicinity of the condensation temperature have been proposed and analysed for decades. We first recall an important early approach, and then develop the scaling law that we have employed to analyse our experiments and simulations.

Non-linear magnetisation effects, and their scaling properties in spin glasses, were first introduced by Malozemoff, Barbara, and Imry [37-39], who introduced the relation for the singular part of the magnetic susceptibility,

$$
\chi_{s}=H^{2 / \delta} f\left(t_{\mathrm{r}} / H^{2 / \phi}\right),
$$

where $f(x)$ is a constant for $x \rightarrow 0 ; f(x)=x^{-\gamma}$ for $x \rightarrow \infty ; \phi=\gamma \delta /(\delta-1) \equiv \beta \delta$; and $t_{\mathrm{r}}$ is the reduced temperature $T / T_{\mathrm{g}}$. This form was used by Lévy and Ogielski [40] and by Lévy [41], who measured the AC non-linear susceptibilities of very dilute AgMn alloys above and below $T_{\mathrm{g}}$ as a function of frequency, temperature, and magnetic field. The critical exponents of equation (18) were evaluated as $\beta=0.9, \gamma=2.3, \delta=3.3, \nu=1.3$, and $z=5.4$. They differ substantially from Monte Carlo simulations for short-range Ising systems: $\beta=0.782(10), \gamma=6.13(11), \nu=2.562(42)$ from [27]. The discrepancy in the value of $\gamma$ is very large, and most probably arises from the lack of an exact value for $T_{\mathrm{g}}$ in the experiments. This illustrates the value of and need for a different approach for scaling the non-linear magnetisation of spin glasses in the vicinity of $T_{\mathrm{g}}$.

Our approach is to express the non-linear components of the magnetic susceptibility in terms of $\xi\left(t, t_{\mathrm{w}}\right)$, the spin-glass correlation length in a magnetic field $H .^{21}$ This approach gives the non-linear magnetisation a direct connection to a measurable quantity and obviates the need for an accurate value of $T_{\mathrm{g}}$.

The argument goes as follows. Let $M\left(t, t_{\mathrm{w}} ; H\right)$ be the magnetisation per spin, where explicit attention is paid to the waiting (ageing) time $t_{\mathrm{w}}$ in the preparation of the spinglass state. The generalised susceptibilities $\chi_{1}, \chi_{3}, \chi_{5}, \ldots$ are defined through the Taylor expansion

$$
M(H)=\chi_{1} H+\frac{\chi_{3}}{3 !} H^{3}+\frac{\chi_{5}}{5 !} H^{5}+\mathcal{O}\left(H^{7}\right) .
$$

where, for brevity's sake, we omit arguments $t$ and $t_{\mathrm{w}}$.

Under equilibrium conditions, and for a large-enough correlation length $\xi_{\text {eq }}$, there is a scaling theory for the magnetic response to an external field $H[42,43]$. Our main hypothesis in this work is that this scaling theory holds not only at equilibrium, but also in the non-equilibrium regime for a spin glass close to $T_{\mathrm{g}}$ and in the presence of a small external magnetic field $H$ :

$$
M\left(t, t_{\mathrm{w}} ; H\right)=\left[\xi\left(t+t_{\mathrm{w}}\right)\right]^{y_{H}-D} \mathcal{F}\left(H\left[\xi\left(t+t_{\mathrm{w}}\right)\right]^{y_{H}}, \frac{\xi\left(t+t_{\mathrm{w}}\right)}{\xi\left(t_{\mathrm{w}}\right)}\right) .
$$

\footnotetext{
${ }^{21}$ The correlation length $\xi\left(t, t_{\mathrm{w}}\right)$ is of course also a function of the temperature $T$, but here we are only interested in the non-linearity of the magnetisation.
} 
According to full-aging spin-glass dynamics (see, e.g. [44]), equation (20) tells us that $\xi\left(t+t_{\mathrm{w}}\right) / \xi\left(t_{\mathrm{w}}\right)$ will be approximately constant close to the maximum of the relaxation rate (see figure 2), so we shall omit this dependence. Hence, combining equations (19) and (20), one can express the generalised susceptibilities $\chi_{1}, \chi_{3}, \chi_{5}, \ldots$ in terms of the spin-glass correlation length $\xi\left(t, t_{\mathrm{w}} ; H\right)$ :

$$
\chi_{2 n-1} \propto\left|\xi\left(t_{\mathrm{w}}\right)\right|^{2 n} y_{H}-D
$$

where we have omitted the arguments $t, H$ for convenience, and

$$
2 y_{H}=D-\frac{\theta(\tilde{x})}{2}
$$

with $\theta(\tilde{x})$ the replicon exponent [23].

The first term of $M(H)$ in equation (19) is $\chi_{1}$, which contains the linear term as well as the first non-linear scaling term [4], so we write

$$
\chi_{1}=\frac{\hat{S}}{T}+\frac{a_{1}(T)}{\xi^{\theta(\tilde{x}) / 2}} .
$$

where $\hat{S}$ is the function appearing in the FDRs [45] and $a_{1}(T)$ is some unknown constant (hopefully smoothly varying with temperature).

The free-energy variation per spin in presence of a magnetic field can be obtained by integrating the magnetic density, equation (19), with respect to the magnetic field:

$$
\Delta F=-\left[\frac{\chi_{1}}{2} H^{2}+\frac{\chi_{3}}{4 !} H^{4}+\frac{\chi_{5}}{6 !} H^{6}+\mathcal{O}\left(H^{8}\right)\right] .
$$

Substituting the scaling behaviour from equations (21) and (23), the free energy $\Delta F$ can be written as (we drop the $\tilde{x}$ dependence of $\theta$ for brevity)

$$
\Delta F=-\left[\frac{\hat{S}}{2 T} H^{2}+\frac{a_{1}(T)}{\xi^{\theta / 2}} H^{2}+a_{3}(T) \xi^{D-\theta} H^{4}+a_{5}(T) \xi^{2 D-(3 \theta / 2)} H^{6}+\mathcal{O}\left(H^{8}\right)\right],
$$

where again the $a_{n}(T)$ are unknowns and (again, hopefully) smoothly varying functions of temperature. We use the effective response time, $t_{H}^{\mathrm{eff}}$, to reflect the total free-energy change at magnetic field $H$ and $H=0^{+}$:

$$
\ln \left[\frac{t_{H}^{\mathrm{eff}}}{t_{H \rightarrow 0^{+}}^{\mathrm{eff}}}\right]=N_{\text {corr }} \Delta F
$$

where $N_{\text {corr }}$ is the number of correlated spins, $N_{\text {corr }}=V_{\text {corr }} / a_{0}^{3}$, with $V_{\text {corr }}$ the correlated spins volume and $a_{0}$ the lattice constant or average distance between magnetic moments. Combining equation (26) with equations (6) and (24) leads to

$$
\begin{aligned}
\ln \left[\frac{t_{H}^{\mathrm{eff}}}{t_{H \rightarrow 0^{+}}^{\mathrm{eff}}}\right]= & -b\left[\left(\frac{\hat{S}}{2 T}+\frac{a_{1}(T)}{\xi^{\theta / 2}}\right) \xi^{D-(\theta / 2)} H^{2}\right. \\
& \left.+a_{3}(T) \xi^{2 D-(3 \theta / 2)} H^{4}+a_{5}(T) \xi^{3 D-2 \theta} H^{6}+\mathcal{O}\left(H^{8}\right)\right],
\end{aligned}
$$


Spin-glass dynamics in the presence of a magnetic field: exploration of microscopic properties

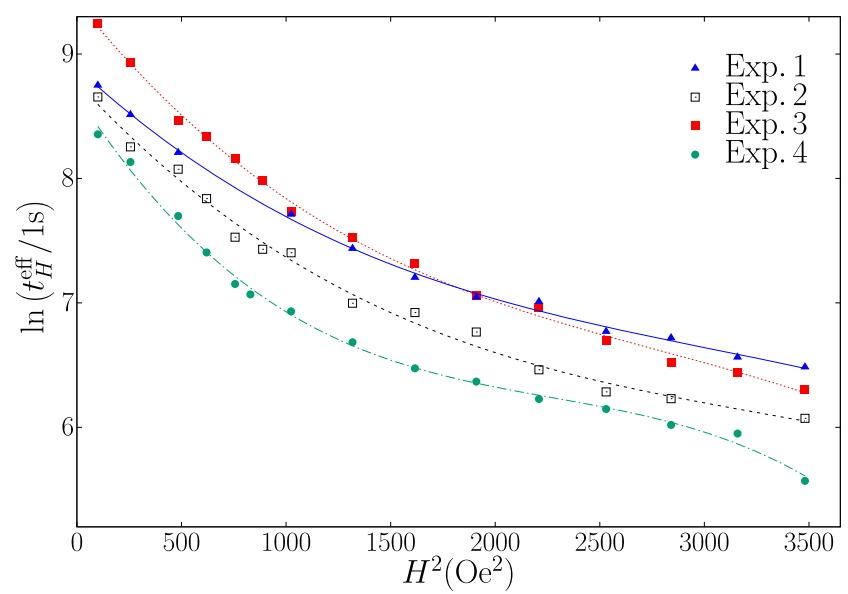

Figure 6. A plot of the peak times $t_{H}^{\mathrm{eff}}$ for the single-crystal CuMn 6 at. $\%$ vs $H^{2}$ for the four values of $T_{\mathrm{m}}$ and $t_{\mathrm{w}}$ listed in table 1 . The slope for small $H^{2}$ is used to extract $\xi\left(t_{\mathrm{w}}\right)$ in table 1 , and the lines come from the fits to the scaling law introduced in section 5.1.

where coefficient $b$ is a geometrical factor, see equation (6), and we have absorbed the $k_{\mathrm{B}} T$ term in the $a_{n}(T)$ coefficients. The correction term $a_{1}(T) / \xi^{\theta(\tilde{x}) / 2}$ is small compared to $\hat{S} / T$, so it will be dropped in subsequent expressions. Equation (27) shows that the higher-order terms have the functional form

$$
\chi_{2 n-1} \frac{H^{2 n}}{(2 n) !}=a_{2 n-1}(T) \xi^{-\theta(\tilde{x}) / 2}\left[\xi^{2 y_{H}} H^{2}\right]^{n}
$$

where

$$
2 y_{H}=D-\frac{\theta(\tilde{x})}{2}
$$

This leads to the new scaling relation,

$$
\ln \left[\frac{t_{H}^{\mathrm{eff}}}{t_{H \rightarrow 0^{+}}^{\mathrm{eff}}}\right]=\frac{\hat{S}}{2 T} \xi^{D-\theta(\tilde{x}) / 2} H^{2}+\xi^{-\theta(\tilde{x}) / 2} \mathcal{G}\left(T, \xi^{D-\theta(\tilde{x}) / 2} H^{2}\right),
$$

where the geometrical factor $b$ has been absorbed into the scaling function $\mathcal{G}$. Comparison with the previous, more classical, relation, equation (18), evinces the simplicity and power of our approach to scale the non-linear magnetisation in the vicinity of $T_{\mathrm{g}}$.

\subsection{Experimental non-linear magnetisation}

We extract the effective waiting time $t_{H}^{\text {eff }}$ in equation (3) from the time at which $S\left(t, t_{\mathrm{w}} ; H\right)$ is a maximum, as before. Our results for all four conditions in table 1 are exhibited as a function of $H^{2}$ in figure 6 . The slope of the data in figure 6 at small values of the magnetic field $H$ generates the spin-glass correlation length $\xi\left(t_{\mathrm{w}} ; T_{\mathrm{m}}\right)$ from equations (4) and (5), see table 1, which also lists the employed values for the replicon 
Spin-glass dynamics in the presence of a magnetic field: exploration of microscopic properties

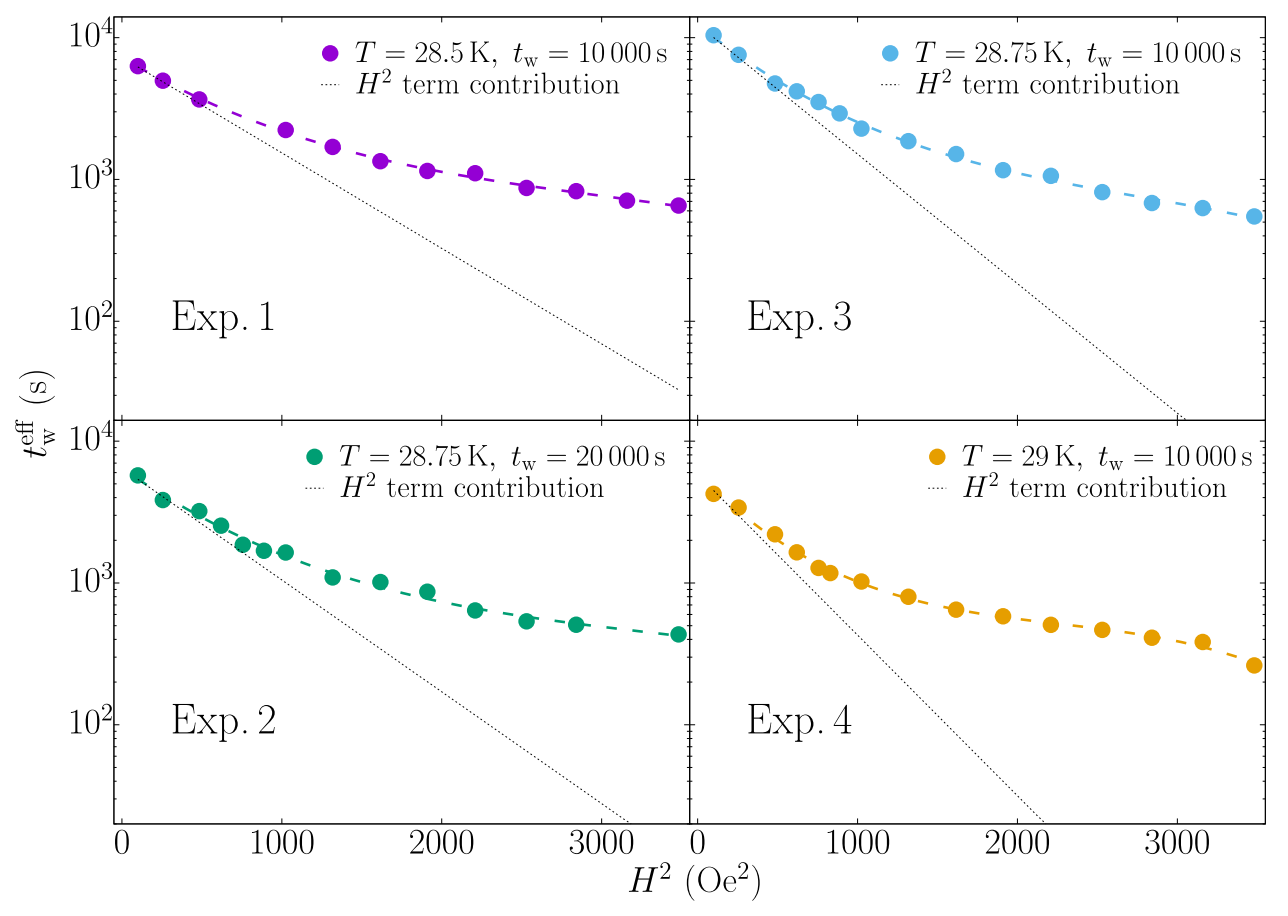

Figure 7. Plots of the peak time $t_{H}^{\text {eff }}$ for the single-crystal CuMn 6 at. $\%$ vs $H^{2}$ for the four experimental regimes of table 1 . The straight lines are extrapolations of the linear term in the magnetisation, and the dashed lines are fits to equation (27).

exponent $\theta(\tilde{x})$. These results will allow us to express the non-linear susceptibility in terms of $\xi\left(t_{\mathrm{w}}\right)$.

An example of the measured relaxation function $S\left(t, t_{\mathrm{w}} ; H\right)$ is plotted for $T_{\mathrm{m}}=28.5 \mathrm{~K}$ and $t_{\mathrm{w}}=10000 \mathrm{~s}$ in figure 2 for five different magnetic fields, while the effective response times, $\ln t_{H}^{\text {eff }}$, are plotted in figure 6 for all four experiments listed in table 1. Note the remarkable similarity in shape of the original experimental results for $\ln t_{H}^{\mathrm{eff}}$ in figure 1 with our results in figure 6 . Also, note the fits of all four of our results for $\ln t_{H}^{\mathrm{eff}}$ in figure 6 to the scaling relationship for the non-linear magnetisation, equation (27), which will be described in more detail below.

Because the scaling relationship, equation (30), depends upon the magnitude of the waiting time in $\xi\left(t, t_{\mathrm{w}} ; T_{\mathrm{m}}\right)$, two different values of $t_{\mathrm{w}}$ were used at the same intermediate temperature $T_{\mathrm{m}}=28.75 \mathrm{~K}$, among the three temperatures $(28.5 \mathrm{~K}, 28.75 \mathrm{~K}$, and $29.0 \mathrm{~K})$ listed in section 2 and in table 1, to test equation (30) at a given temperature. This allows us to discriminate between the influence of temperature and of waiting time on $\xi\left(t, t_{\mathrm{w}} ; T_{\mathrm{m}}\right)$. In this way, we are able to demonstrate explicitly that $\xi\left(t, t_{\mathrm{w}} ; T_{\mathrm{m}}\right)$ is the control parameter.

It is useful to display $t_{H}^{\mathrm{eff}}$ against $H^{2}$ individually for each of the four values of $T_{\mathrm{m}}$ and $t_{\mathrm{w}}$. They are exhibited above in figure 7 . The data for $\ln t_{H}^{\mathrm{eff}}$ is fitted to the function

$$
f(x)=c_{0}+c_{2} x+c_{4} x^{2}+c_{6} x^{3}+\mathcal{O}\left(x^{4}\right),
$$


Spin-glass dynamics in the presence of a magnetic field: exploration of microscopic properties

Table 3. Parameters from our fits to equation (31) of our experimental data for $\ln t_{H}^{\mathrm{eff}}$, as a function of $T_{\mathrm{m}}$ and $t_{\mathrm{w}}$ (data from figure 7 ). The uninteresting fit parameter $c_{0}$ is not included in the table.

\begin{tabular}{lccc}
\hline$T_{\mathrm{m}}(\mathrm{K})$ & $t_{\mathrm{w}}(\mathrm{s})$ & Coefficient & Numerical value \\
\hline \multirow{2}{*}{28.5} & \multirow{3}{*}{10000} & $c_{2}$ & $-1.55(10) \times 10^{-3}$ \\
& & $c_{4}$ & $4.0(7) \times 10^{-7}$ \\
& & $c_{6}$ & $-4.4(13) \times 10^{-11}$ \\
\hline \multirow{3}{*}{28.75} & \multirow{2}{*}{10000} & $c_{2}$ & $-1.82(20) \times 10^{-3}$ \\
& & $c_{4}$ & $4.6(13) \times 10^{-7}$ \\
& & $c_{6}$ & $-4.6(25) \times 10^{-11}$ \\
\hline \multirow{2}{*}{28.75} & \multirow{2}{*}{20000} & $c_{2}$ & $-2.10(12) \times 10^{-3}$ \\
& & $c_{4}$ & $5.9(8) \times 10^{-7}$ \\
& & $c_{6}$ & $-7.0(15) \times 10^{-11}$ \\
\hline \multirow{2}{*}{29} & \multirow{2}{*}{10000} & $c_{2}$ & $-2.61(13) \times 10^{-3}$ \\
& & $c_{4}$ & $1.02(8) \times 10^{-6}$ \\
& & $c_{6}$ & $-1.49(16) \times 10^{-10}$ \\
\hline
\end{tabular}

where $x \equiv H^{2}$ and the $c_{n}$ coefficients, according to equation (27), correspond to:

$$
\begin{aligned}
& c_{0}=\ln \left(t_{H \rightarrow 0^{+}}^{\mathrm{eff}}\right), \\
& c_{2}=\left[\frac{\hat{S}}{2 T_{\mathrm{m}}}\right] \xi^{D-\theta(\tilde{x}) / 2}, \\
& c_{4}=a_{3}\left(T_{\mathrm{m}}\right) \xi^{2 D-3 \theta(\tilde{x}) / 2}, \\
& c_{6}=a_{5}\left(T_{\mathrm{m}}\right) \xi^{3 D-2 \theta(\tilde{x})} .
\end{aligned}
$$

Notice that we have absorbed the geometrical prefactor $b$ of equation (27) in the nonlinear coefficients $a_{n}\left(T_{\mathrm{m}}\right)$ and in the linear coefficient $\hat{S}$, and we neglect the sub-leading coefficient $a_{1}\left(T_{\mathrm{m}}\right) / \xi^{\theta(\tilde{x}) / 2}$.

The effect of increasing temperature with waiting time held constant can be seen in the difference between the measured $t_{H}^{\mathrm{eff}}$ and the extrapolated value of the linear magnetisation term (quadratic in $\left.H^{2}\right)$ for the largest magnetic field $(H=59 \mathrm{Oe})$ in exps. 1, 2 and 4 in figure 7. Non-linear effects grow for larger $t_{\mathrm{w}}$, hence larger $\xi\left(t, t_{\mathrm{w}} ; T_{\mathrm{m}}\right)$ at the same temperature, which can be seen by comparing experiments 2 and 3 . The linear and non-linear coefficients of equation (27) can be extracted from fits of the data in figure 7 (dashed lines), whose resulting coefficients are listed in table 3.

To test the scaling relationship of equation (30) we first consider the fits of the data at $T_{\mathrm{m}}=28.75 \mathrm{~K}$ for the two waiting times, $t_{\mathrm{w}}=2 \times 10^{4} \mathrm{~s}$ and $t_{\mathrm{w}}=10^{4} \mathrm{~s}$. The linear term is proportional to $\xi^{D-\theta(\tilde{x}) / 2}$. The ratio of the two correlation lengths from table 3 is, hence,

$$
\frac{\xi\left(t_{\mathrm{w}}=20000 \mathrm{~s}\right)}{\xi\left(t_{\mathrm{w}}=10000 \mathrm{~s}\right)}=\left[\frac{c_{2}\left(t_{\mathrm{w}}=20000 \mathrm{~s}\right)}{c_{2}\left(t_{\mathrm{w}}=10000 \mathrm{~s}\right)}\right]^{1 /[D-\theta(\tilde{x}) / 2]} \approx 1.0535 .
$$


Spin-glass dynamics in the presence of a magnetic field: exploration of microscopic properties

Should scaling hold according to equation (30), then consistency requires that the ratios of the correlation lengths from the non-linear terms be the same as that for the linear term. They are:

$$
\frac{\xi\left(t_{\mathrm{w}}=20000 \mathrm{~s}\right)}{\xi\left(t_{\mathrm{w}}=10000 \mathrm{~s}\right)}=\left[\frac{c_{4}\left(t_{\mathrm{w}}=20000 \mathrm{~s}\right)}{c_{4}\left(t_{\mathrm{w}}=10000 \mathrm{~s}\right)}\right]^{1 /[2 D-3 \theta(\tilde{x}) / 2]} \approx 1.0476,
$$

and

$$
\frac{\xi\left(t_{\mathrm{w}}=20000 \mathrm{~s}\right)}{\xi\left(t_{\mathrm{w}}=10000 \mathrm{~s}\right)}=\left[\frac{c_{6}\left(t_{\mathrm{w}}=20000 \mathrm{~s}\right)}{c_{6}\left(t_{\mathrm{w}}=10000 \mathrm{~s}\right)}\right]^{1 /[3 D-2 \theta(\tilde{x})]} \approx 1.0526
$$

The equality (within experimental error) of equations (36)-(38) is an impressive experimental verification of scaling relationship (30). Another check is the growth of the correlation length itself. At temperature $T_{\mathrm{m}}=28.75 \mathrm{~K}$ and for the two waiting times, it is possible to calculate the ratio of the two values of the correlation length directly, using the expression for power-law growth $[5,46]$,

$$
\xi\left(t_{\mathrm{w}} ; T_{\mathrm{m}}\right)=a_{0} \hat{C}_{1}\left(\frac{t_{\mathrm{w}}}{\tau_{0}}\right)^{\hat{C}_{2}\left(T_{\mathrm{m}} / T_{\mathrm{g}}\right)} \equiv a_{0} \hat{C}_{1}\left(\frac{t_{\mathrm{w}}}{\tau_{0}}\right)^{T_{\mathrm{m}} /\left(z_{\mathrm{c}} T_{\mathrm{g}}\right)},
$$

where $\hat{C}_{1}$ and $\hat{C}_{2}$ are constants, by definition $\hat{C}_{2} \equiv 1 / z_{\mathrm{c}}$, and $\tau_{0}$ is a characteristic exchange time, here taken as $\hbar / k_{\mathrm{B}} T_{\mathrm{g}}$.

Using the growth-rate parameter $z_{\mathrm{c}}=12.37(107)[2,3]$ one finds

$$
\frac{\xi\left(t_{\mathrm{w}}=20000 \mathrm{~s}\right)}{\xi\left(t_{\mathrm{w}}=10000 \mathrm{~s}\right)} \approx\left(\frac{2 \times 10^{4}}{10^{4}}\right)^{T_{\mathrm{m}} /\left(12.37 T_{\mathrm{g}}\right)}=2^{28.75 /(12.37 \times 31.5)} \approx 1.0525 .
$$

Comparing the ratio of $\xi\left(t_{\mathrm{w}} ; T_{\mathrm{m}}\right)$ for the two different waiting times, equation (40), from the growth law, equation (39), with the ratios from fitting to the scaling relationship, equations (36)-(38), is remarkable evidence for the consistency of our physical picture. It explicitly demonstrates the power of using the spin-glass correlation length as the primary factor for evaluating the spin-glass non-linear magnetisation in the vicinity of the transition temperature $T_{\mathrm{g}}$.

The lingering issue from equations (6) and (30), according to Zhai et al [3] is that the replicon exponent $[\theta(\tilde{x})](\ldots)$ depends upon both the temperature and $\xi$ through the crossover variable $[\tilde{x}]^{\prime}$, with

$$
\tilde{x}=\frac{\ell_{\mathrm{J}}}{\xi\left(t_{\mathrm{w}} ; T_{\mathrm{m}}\right)} .
$$

From the notation of table 3 and equation (6) the number of correlated spins is

$$
N_{\text {corr }}=\frac{k_{\mathrm{B}} T_{\mathrm{m}} c_{2}}{\chi_{\mathrm{FC}}}=\xi^{D-\theta(\tilde{x}) / 2}=\xi^{D-\left[\theta\left(\ell_{\mathrm{J}} / \xi\right) / 2\right]} .
$$

The left-hand side is a number, the right-hand side is an implicit function of $\xi$ and $\theta$. Using the definition of the Josephson length $\ell_{\mathrm{J}}$ and of the replicon $\theta(\tilde{x})^{22}$, one can solve

22 The reader will find all the necessary details in appendix B. 
Spin-glass dynamics in the presence of a magnetic field: exploration of microscopic properties

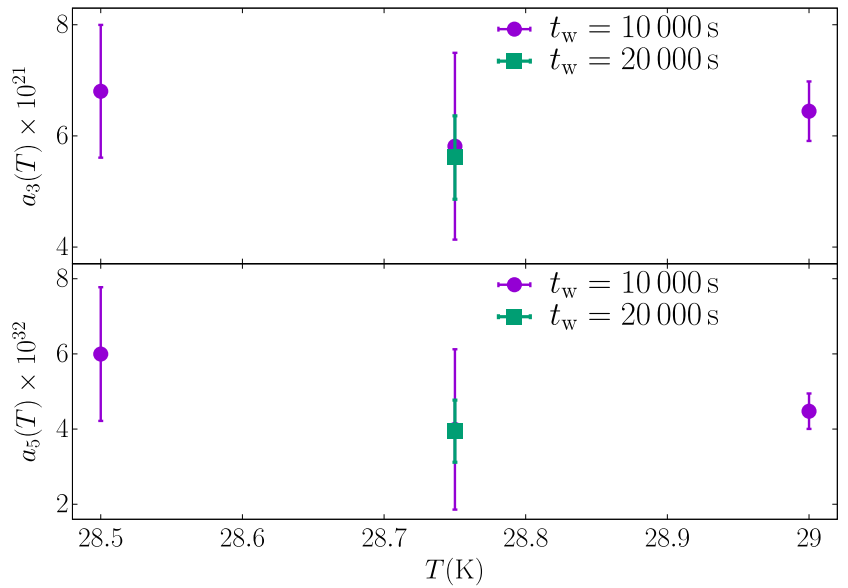

Figure 8. Non-linear coefficients $a_{3}$ and $a_{5}$, as defined in equation (27), calculated using the extracted values of $\xi$ and $\theta$ for the different measuring temperatures $T_{\mathrm{m}}$ and waiting times $t_{\mathrm{w}}$ in our experiments.

for $\xi$ and $\theta$ at each of the four values of $T_{\mathrm{m}}$ and $t_{\mathrm{w}}$ explored experimentally. The results are displayed in table 1.

The ageing rate $z_{\mathrm{c}}$ varies as a function of $\xi$. Using the data at $T_{\mathrm{m}}=28.75 \mathrm{~K}$, the approximate aging-rate factor is $z_{\mathrm{c}}=12.37(107)$ at $\xi \sim 200$ lattice spacings $a_{0}[3]$. Although the correlation length extracted at $28.75 \mathrm{~K}$ is larger than that at $28.5 \mathrm{~K}$, the higher temperature sets the crossover variable $x=\ell_{\mathrm{J}}\left(T_{\mathrm{m}}\right) / \xi=0.11$ for $t_{\mathrm{w}}=20000$ $\mathrm{s}$ and $x=0.12$ for $t_{\mathrm{w}}=10000 \mathrm{~s}$, in the range of the crossover variable obtained by us previously at $T_{\mathrm{m}}=28.5 \mathrm{~K}$ [47]. Both measurements have exhibited slowing down of the spin-glass correlation growth rate near the critical temperature at large correlation lengths.

Using the average value of $\theta$ from table $1, \theta=0.343$, and setting $z_{\mathrm{c}}=12.37$, the values exhibited in equations (36)-(40) are altered to 1.053, 1.048, 1.052, and 1.051, respectively. Using the values from table 1 , the temperature-dependent coefficients $a_{3}\left(T_{\mathrm{m}}\right)$ and $a_{5}\left(T_{\mathrm{m}}\right)$ of equation (27) can be calculated for each of the four values of $T_{\mathrm{m}}$ and $t_{\mathrm{w}}$. They are displayed in figure 8.

From figure 8, one sees that the 'hope' expressed after equation (25), i.e. that the temperature dependence of the coefficients $a_{n}\left(T_{\mathrm{m}}\right)$ appearing in equation (25) be weak, is realised in this set of experiments. For $a_{3}\left(T_{\mathrm{m}}\right)$, within the experimental error bars, there is little or no change with temperature. The situation for $a_{5}\left(T_{\mathrm{m}}\right)$ is not as nice, but there appears to be little change with temperature at the two highest temperatures.

It is interesting to test the scaling relationship [4]

$$
\chi_{2 n-1}\left(t_{\mathrm{w}} ; T_{\mathrm{m}}\right) \propto a_{2 n-1}\left[\xi\left(t_{\mathrm{w}} ; T_{\mathrm{m}}\right)\right]^{(n-1) D-n \theta(\tilde{x}) / 2} .
$$

Thus,

$$
\chi_{3} \propto \xi^{D-\theta(\tilde{x})} a_{3}, \quad \chi_{5} \propto \xi^{2 D-\frac{3 \theta(\tilde{x})}{2}} a_{5} .
$$


Spin-glass dynamics in the presence of a magnetic field: exploration of microscopic properties

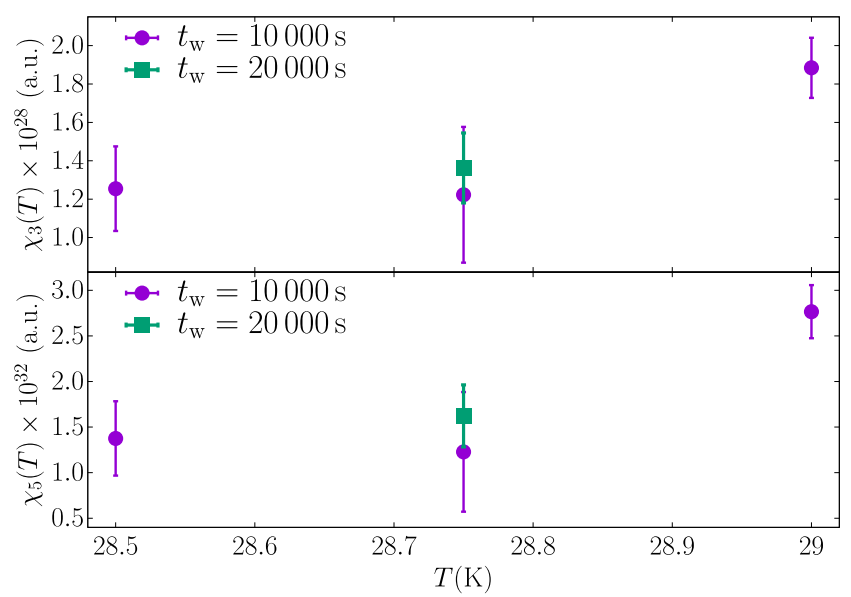

Figure 9. Non-linear susceptibilities $\chi_{3}\left(t_{\mathrm{w}} ; T_{\mathrm{m}}\right)$ and $\chi_{5}\left(t_{\mathrm{w}} ; T_{\mathrm{m}}\right)$ from equation (42), plotted as a function of temperature for the four experimental regimes of table 1 .

The measured non-linear susceptibilities are exhibited below for the three temperatures $28.5 \mathrm{~K}, 28.75 \mathrm{~K}$ and $29.0 \mathrm{~K}$.

One can test the scaling relationships (43) and (44) by using the measured values for the spin-glass correlation length $\xi$, the replicon exponent $\theta(\tilde{x})$ from table 1 , and the values of $c_{2}$ and $c_{4}$ from temperatures $28.5 \mathrm{~K}$ and $29 \mathrm{~K}$ and $t_{\mathrm{w}}=10000 \mathrm{~s}$. For $T_{\mathrm{m}}=28.5 \mathrm{~K}$, we have $\xi=320.36 a_{0}, \theta(\tilde{x})=0.337$ (from table 1 ), and $c_{4}=4.0 \times 10^{-7}$ (from table 3 , note that we have ignored the error bars). Similarly, for $T_{\mathrm{m}}=29.0 \mathrm{~K}$ and $t_{\mathrm{w}}=10000 \mathrm{~s}$ we have $\xi=391.27 a_{0}, \theta(\tilde{x})=0.349$, and $c_{4}=10.2 \times 10^{-7}$. Using equations (31) and (34) and the just quoted values of $c_{4}\left(t_{\mathrm{w}} ; T_{\mathrm{m}}\right)$ one finds

$$
\frac{\chi_{3}\left(t_{\mathrm{w}}=10000 \mathrm{~s} ; T_{\mathrm{m}}=28.5 \mathrm{~K}\right)}{\chi_{3}\left(t_{\mathrm{w}}=10000 \mathrm{~s} ; T_{\mathrm{m}}=29.0 \mathrm{~K}\right)} \approx 0.666 .
$$

This ratio is well within the error bars of the measured non-linear susceptibilities in figure 9. A similar result is also found for $\chi_{5}$.

With these scaling observations in hand, it is interesting to wonder about using them to estimate the condensation temperature $T_{\mathrm{g}}$. In principle, determination of $T_{\mathrm{g}}$ would require an infinite $t_{\mathrm{w}}$, because $\xi\left(T_{\mathrm{m}}\right) \rightarrow \infty$ when $T_{\mathrm{m}} \rightarrow T_{\mathrm{g}}$. One expects that any experiment at finite $t_{\mathrm{w}}$ would yield a maximum for the non-linear susceptibility at a temperature we shall call $T_{\mathrm{g}}\left(t_{\mathrm{w}}\right)$ because $t_{\mathrm{w}}$ is finite.

In principle, then, by measuring $T_{\mathrm{g}}\left(t_{\mathrm{w}}\right)$ for ever larger $t_{\mathrm{w}}$, one could extrapolate to the true $t_{\mathrm{w}} \rightarrow \infty$ condensation temperature $T_{\mathrm{g}}$. If nothing else, measurements at large values of $t_{\mathrm{w}}$ on laboratory time scales could establish a lower bound for $T_{\mathrm{g}}$.

The non-linear susceptibility $\chi_{3}$ diverges as

$$
\chi_{3}\left(t_{\mathrm{w}} \rightarrow \infty ; T_{\mathrm{m}}\right)=\chi_{0} \frac{T_{\mathrm{g}}\left(t_{\mathrm{w}} \rightarrow \infty\right)}{\left|T_{\mathrm{g}}\left(t_{\mathrm{w}} \rightarrow \infty\right)-T_{\mathrm{m}}\right|^{\gamma}},
$$

where $\chi_{0}$ is a constant independent of temperature, and $\gamma=6.13(11)$ [27]. For finite $t_{\mathrm{w}}$, $\chi_{3}\left(t_{\mathrm{w}} ; T_{\mathrm{m}}\right)$ only has a maximum as a function of temperature. A way of arriving at this 
maximum would be to fit the data to the function

$$
\chi_{3}\left(t_{\mathrm{w}} ; T_{\mathrm{m}}\right)=\chi_{0} \frac{T_{\mathrm{g}}\left(t_{\mathrm{w}}\right)}{\left|T_{\mathrm{g}}\left(t_{\mathrm{w}}\right)-T_{\mathrm{m}}\right|^{\gamma}},
$$

and then use the data points from just two or three temperatures to extract $T_{\mathrm{g}}\left(t_{\mathrm{w}}\right)$. For larger and larger $t_{\mathrm{w}}$, one could in principle extrapolate to the true $T_{\mathrm{g}}$. We emphasise that, though equation (47) suggests $\chi_{3}\left(t_{\mathrm{w}} ; T_{\mathrm{m}}\right)$ diverges at $T_{\mathrm{m}}=T_{\mathrm{g}}\left(t_{\mathrm{w}}\right)$, it does not, arriving only at a maximum value for finite $t_{\mathrm{w}}$. Nevertheless, equation (47) is a way of estimating $T_{\mathrm{g}}\left(t_{\mathrm{w}}\right)$ for use in an extrapolation procedure.

To test whether this trick has any validity, consider the data exhibited in figure 9 . Here, $t_{\mathrm{w}}=10000 \mathrm{~s}$ and, taking $\chi_{3}\left(t_{\mathrm{w}} ; T_{\mathrm{m}}\right)$ at the centre of the error bars for the two temperatures $28.5 \mathrm{~K}$ and $29 \mathrm{~K}$, one finds $T_{\mathrm{g}}\left(t_{\mathrm{w}}=10000 \mathrm{~s}\right)=32 \mathrm{~K}$. This value is too high, as magnetisation measurements suggest $T_{\mathrm{g}}\left(t_{\mathrm{w}} \rightarrow \infty\right)=31.5 \mathrm{~K}$. More accurate determination of the parameters in table 3 would diminish the error in $T_{\mathrm{g}}\left(t_{\mathrm{w}}\right)$, but it does suggest a feasible process for taking laboratory data for finite $t_{\mathrm{w}}$ and extrapolating to find $T_{\mathrm{g}}\left(t_{\mathrm{w}} \rightarrow \infty\right)$.

\subsection{Reanalysis of previous data}

Given the above analysis of our recent data, it is convenient to revisit the work of Joh et al [5] and of Bert et al [9], to examine whether the Zeeman energy is proportional to $H^{2}$ or to $H$ (alternatively, to the number of correlated spins or to the square root of the total number of spins, respectively). We have already alluded to the results of these works as displaying the effect of magnetisation non-linearity. We now explore this assertion in detail using the analysis of subsection 5.2.

Figure 1 of Joh et al and figure 3 of Bert et al are reproduced in figures 1 and 10 in this paper. Both exhibit significant deviations from an $H^{2}$ dependence of the $\ln t_{H}^{\mathrm{eff}}$ with increasing values of $H$. Bert et al [9] go on to assert a linear dependence, as exhibited in their figure 3, reproduced here in figure 10. The magnetic fields in [9] are quite large, and the scale of their plot does not cover the dependence on $H^{2}$ for small $H$. Nevertheless, they claim their data fits a linear dependence of $\ln t_{H}^{\mathrm{eff}}$ on $H$. A glance at the left panel of figure 10 suggests how they could rationalise their conclusion.

Yet, as noted by the authors of the experiments in figure 1 [5], a linear dependence on $H$ is a poor fit to the data at small $H$. Further, the argument for the magnetisation's growth with $\sqrt{N_{\text {corr }}}$ is supposedly valid at small $H$ [48], while the dependence on the number of correlated spins is argued to be proportional to $\sqrt{N_{\text {corr }}}$, rather than linear in $N_{\text {corr }}$, as from equation (4). On the other hand, the data exhibited in figure 10 uses magnetic fields that are substantially larger than those considered in our experiments.

We assert that the departure from linearity in $H^{2}$ as $H$ increases observed in [9] is simply the effect of non-linearity. To prove this, we apply the scaling relation, equation (30), to their data, doing our best to extract their measured values from their figure. Our fit to equation (31) is shown in the right panel of figure 10 and the resulting $c_{n}$ are listed in table 4.

Although only 1-2 digits are significant in table 4, we write more digits, for the sake of reproducibility. The fitting quality for $t_{\mathrm{w}}=10000 \mathrm{~s}$ and $t_{\mathrm{w}}=30000 \mathrm{~s}$ is better 
Spin-glass dynamics in the presence of a magnetic field: exploration of microscopic properties

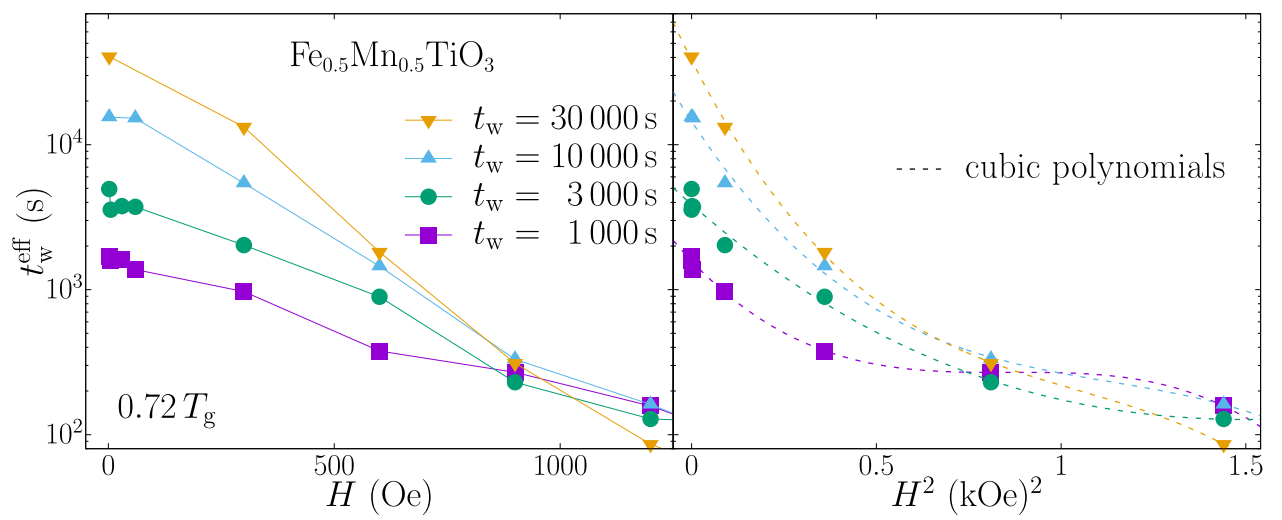

Figure 10. (Left) Effective waiting times (in log scale) derived from field-change experiments on an Ising sample $\left(\mathrm{Fe}_{0.5} \mathrm{Mn}_{0.5} \mathrm{TiO}_{3}\right)$ as a function of the magnetic field $H$. The plot reproduces figure 3 of Bert et al [9] (solid lines are linear interpolations to data with same $t_{\mathrm{w}}$ ). (Right) The same data plotted against $H^{2}$. The dashed lines are fits to equation (31), with fit parameters listed in table 4.

Table 4. We report, as a function of $t_{\mathrm{w}}$, the parameters from fits to equation (31) of the data obtained by Bert et al [9] for $\ln t_{\mathrm{H}}^{\mathrm{eff}}$. Their data correspond to $\mathrm{Fe}_{0.5} \mathrm{Mn}_{0.5} \mathrm{TiO}_{3}$ at $T_{\mathrm{m}}=0.72 T_{\mathrm{g}}$ (see figure 3 of [9]). The fits are shown in our figure 10. The uninteresting fit parameter, $c_{0}$, is not included in the table.

\begin{tabular}{ccc}
\hline$t_{\mathrm{w}}(\mathrm{s})$ & Coefficient & Value \\
\hline \multirow{3}{*}{1000} & $c_{2}$ & $-6.184 \times 10^{-6}$ \\
& $c_{4}$ & $7.193 \times 10^{-12}$ \\
& $c_{6}$ & $-2.784 \times 10^{-18}$ \\
\hline \multirow{3}{*}{3000} & $c_{2}$ & $-5.154 \times 10^{-6}$ \\
& $c_{4}$ & $2.322 \times 10^{-12}$ \\
& $c_{6}$ & $-0.269 \times 10^{-18}$ \\
\hline \multirow{3}{*}{10000} & $c_{2}$ & $-8.977 \times 10^{-6}$ \\
& $c_{4}$ & $7.052 \times 10^{-12}$ \\
& $c_{6}$ & $-2.075 \times 10^{-18}$ \\
\hline \multirow{3}{*}{30000} & $c_{2}$ & $-11.600 \times 10^{-6}$ \\
& $c_{4}$ & $9.440 \times 10^{-12}$ \\
& $c_{6}$ & $-3.004 \times 10^{-18}$ \\
\hline
\end{tabular}

than for the other two waiting times. Note that the coefficients $c_{n}$ listed in table 4 are considerably smaller than in our table 3 for our current experiments on a CuMn 6 at. $\%$ single crystal. We believe this is because our measurements are for $T_{\mathrm{m}} \approx 0.9 T_{\mathrm{g}}$ whereas Bert et al [9] worked at $0.72 T_{\mathrm{g}}$, where non-linear terms are expected to be much smaller.

Using the fitting coefficients from table 4 and $\theta(\tilde{x})=0.3$, we obtain

$$
\frac{\xi\left(t_{\mathrm{w}}=30000 \mathrm{~s}\right)}{\xi\left(t_{\mathrm{w}}=10000 \mathrm{~s}\right)}=\left[\frac{c_{2}(30000 \mathrm{~s})}{c_{2}(10000 \mathrm{~s})}\right]^{1 /[D-\theta(\tilde{x}) / 2]} \approx 1.094
$$


Spin-glass dynamics in the presence of a magnetic field: exploration of microscopic properties

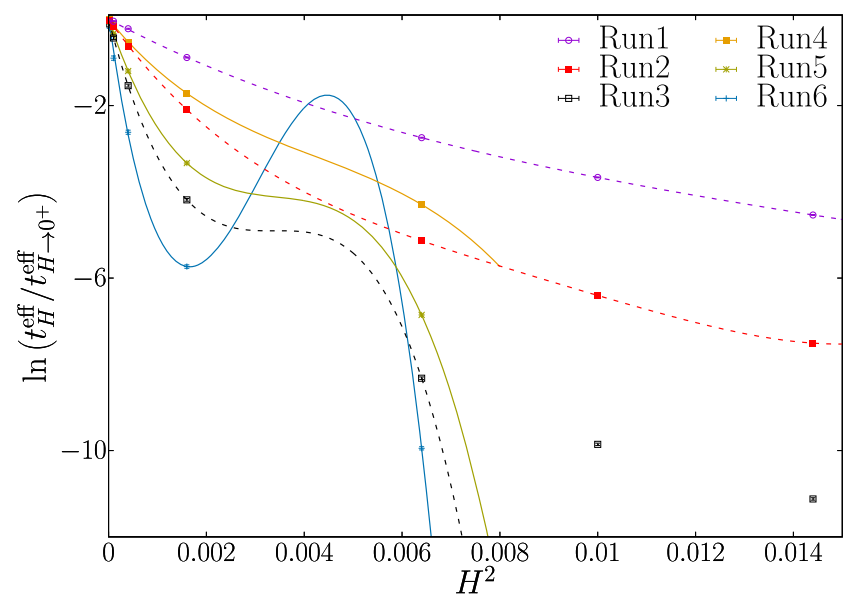

Figure 11. The numerical time ratio $\ln \left(t_{H}^{\mathrm{eff}} / t_{H \rightarrow 0^{+}}^{\mathrm{eff}}\right)$ for the six runs of table 2 . The data were fitted as a polynomial of $H^{2}$ as reported in table 5. Continuous lines are fits for data at $T=1.0$; dashed lines correspond to the data at $T=0.9$.

$$
\begin{aligned}
& \frac{\xi\left(t_{\mathrm{w}}=30000 \mathrm{~s}\right)}{\xi\left(t_{\mathrm{w}}=10000 \mathrm{~s}\right)}=\left[\frac{c_{4}(30000 \mathrm{~s})}{c_{4}(10000 \mathrm{~s})}\right]^{1 /[2 D-3 \theta(\tilde{x}) / 2]} \approx 1.054 \\
& \frac{\xi\left(t_{\mathrm{w}}=30000 \mathrm{~s}\right)}{\xi\left(t_{\mathrm{w}}=10000 \mathrm{~s}\right)}=\left[\frac{c_{6}(30000 \mathrm{~s})}{c_{6}(10000 \mathrm{~s})}\right]^{1 /[3 D-2 \theta(\tilde{x})]} \approx 1.045 .
\end{aligned}
$$

The three ratios, equations (48)-(50) do not agree with one another perfectly, but again, considering the rawness of the analysis, they are certainly suggestive. In summary, we believe that the assessment of [9] that their data is evidence for $E_{\mathrm{Z}} \propto H$ is in error. Rather, we believe the departure they observe from linearity in $H^{2}$ arises from non-linear terms in the magnetisation as a result of the large magnetic fields utilised in their study.

\subsection{Numerical study of the ratio of the effective times at $\boldsymbol{H}$ and $\boldsymbol{H}=\mathbf{O}^{+}$}

We have exploited our proposed relation (16) to extract effective times $t_{H}^{\mathrm{eff}}$, as explained in appendix A.3. Our results are displayed in figure 11. In the subsequent analysis in section 5.5, we shall need the derivative of $\ln \left(t_{H}^{\text {eff }} / t_{H \rightarrow 0+}^{\text {eff }}\right)$ with respect to $H^{2}$, evaluated numerically at $H^{2}=0$. Our main scope here will be evaluating this derivative.

An obvious strategy would be to fit the numerical data for $\ln \left(t_{H}^{\mathrm{eff}} / t_{H \rightarrow 0+}^{\mathrm{eff}}\right)$ as we did for the experimental data in equation (31). Note that our sought derivative at $H^{2}=0$ is just the $c_{2}$ coefficient in the fit. A welcome simplification in the analysis of the numerical data is that we can explicitly put $c_{0}=0$ in the fit to equation (31) (indeed, we are able to carry out the fit for $\ln \left(t_{H}^{\mathrm{eff}} / t_{H \rightarrow 0+}^{\mathrm{eff}}\right)$ thanks to equation (16)). Our fitting parameters are reported in table 5. Unfortunately, as the reader will note from figure 11, these fits predict unphysically wild oscillations that are not observed in the numerical data. A plausible explanation for these oscillations relies on the very large magnetic fields used 
Spin-glass dynamics in the presence of a magnetic field: exploration of microscopic properties

Table 5. Results of the fits to equation (31) of the numerical data for the time ratio $\ln \left(t_{H}^{\mathrm{eff}} / t_{H \rightarrow 0^{+}}^{\mathrm{eff}}\right)$. Note that, in order to stabilise the fits, we needed to include an extra terms in equation (31) for two cases. In the table, $\xi$ stands for $\xi\left(t=0, t_{\mathrm{w}} ; H=0\right)$ and the fitting range is $0 \leqslant H^{2} \leqslant H_{\max }^{2}$.

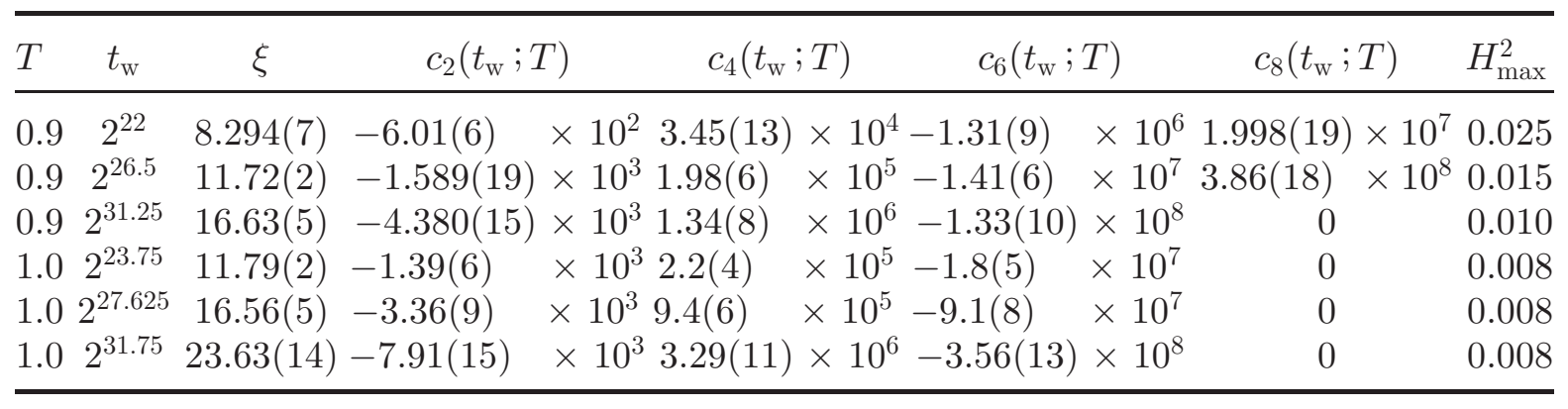

(recall that $H=1$ for the IEA model roughly corresponds to $5 \times 10^{4}$ Oe in physical units). These huge magnetic fields probably exceed the radius of convergence of the Taylor expansion of equation (30). At any rate, the oscillations cast some doubts on the determination of the derivative at $H^{2}=0$. This is why we have turned to a different strategy in order to validate our computation.

Our starting point, recall [2] and equation (27), is the expected scaling behaviour for the coefficient $c_{2}\left(t_{\mathrm{w}} ; T\right)$ listed in table 5 . The non-linear coefficient $c_{2}\left(t_{\mathrm{w}} ; T\right)$, reported in table 5 , behaves as [4]

$$
c_{2}\left(t_{\mathrm{w}} ; T\right)=\xi^{D-\theta(\tilde{x}) / 2}\left(\frac{\hat{S}}{2 T}+\frac{a_{1}(T)}{\xi^{\theta(\tilde{x}) / 2}}\right),
$$

using the scaling of the susceptibility $\chi_{1}$ from equation (23). Here, $\hat{S}$ is the function appearing in the FDR [45] and $a_{1}(T)$ is a smooth function of temperature, and we have absorbed the geometrical prefactor $b$ of equation $(27)$ in $a_{1}(T)$ and $\hat{S}(T)$. Notice that the $a_{1}(T) \xi^{-\theta(\tilde{x}) / 2}$ term is sub-leading compared to $\hat{S} /(2 T)$ and it was neglected in the previous analysis.

We rewrite equation (51) as:

$$
\frac{c_{2}\left(t_{\mathrm{w}} ; T\right)}{\xi^{D-\theta(\tilde{x}) / 2}}=\frac{\hat{S}}{2}+T a_{1}(T) \xi^{-\theta(\tilde{x}) / 2},
$$

and we study this quantity as a function of $\left[\xi\left(t_{\mathrm{w}}\right)\right]^{-\theta(\tilde{x}) / 2}$ in figure 12 . Note that in the above expression $\xi$ was not obtained from the response to the magnetic field. Instead, we computed $\xi$ from the correlation functions at $H=0$ (see appendix A.5 and [7]). The data exhibit a constant value, except for the point correspondent to $t_{\mathrm{w}}=2^{31.75}$ at $T=1.0$ (run 6). Therefore, we shall accept the numerical estimation of the derivative at $H^{2}=0$ through the coefficient $c_{2}$ for all cases but for our run 6 . In order to clarify what is going on with run 6 , we report in the right panel of figure 12 an enlargement of figure 11 in the small-magnetic-field regime for this case. As it could be guessed from the left panel, the fitting procedure clearly underestimates the slope of the curve at $H^{2}=0$. Therefore, in order to estimate the derivative for run 6 , we have instead relied 
Spin-glass dynamics in the presence of a magnetic field: exploration of microscopic properties
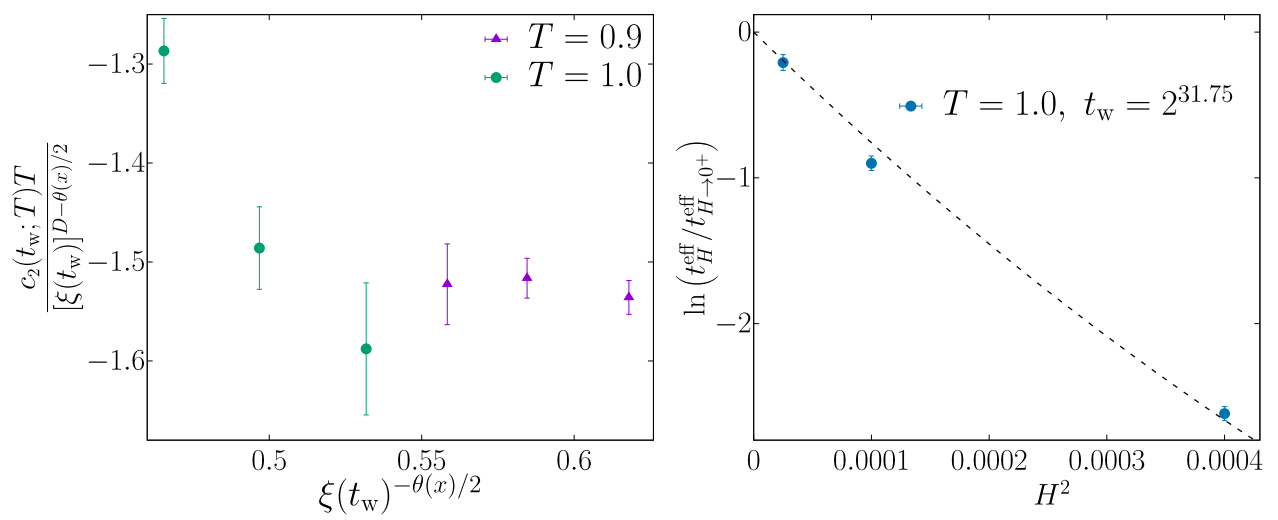

Figure 12. (Left) Behaviour of the rescaled quantity $c_{2}\left(t_{\mathrm{w}} ; T\right) T /\left[\xi\left(t_{\mathrm{w}}\right)\right]^{D-\theta(\tilde{x}) / 2}$ as a function of $\xi\left(t_{\mathrm{w}}\right)^{-\theta(\tilde{x}) / 2}$, see equation (52). (Right) An enlargement of figure 11 in the small-field regime, for the case $t_{\mathrm{w}}=2^{31.75}$ at $T=1.0$ (run 6 of table 2 ) and its fit reported in table 5 .

on equation (52) by averaging the constant value found in figure 12 over all other runs and by multiplying this averaged constant value by $\left[\xi\left(t_{\mathrm{w}}\right)\right]^{D-\theta(\tilde{x}) / 2}$ (see equation $(52)$ ).

\subsection{Non-linear scaling}

In order to test the scaling form, equation (30), the data for all of the non-linear contributions to the magnetisation, experimental and numerical, are plotted according to the functional form

$$
\xi^{-\frac{\theta(\tilde{x})}{2}} \mathcal{G}\left(T, \xi^{D-\frac{\theta(\tilde{x})}{2}} H^{2}\right)
$$

in figures 13 and 14. The fit to scaling relationship (30) is remarkable and testimony to the agreement for both the experimental and numerical data.

\subsection{Overshooting phenomena}

We first address the dynamical scaling law for a system in presence of a magnetic field at temperatures close to $T_{\mathrm{g}}$ in section 5.6.1. Then, in section 5.6.2, we analyse the dynamical scaling for ferromagnetic systems, either Ising or Heisenberg, in the presence of an external magnetic field.

5.6.1. Dynamical scaling close to $T_{\mathrm{g}}$. We evaluate the growth of the correlation length $\xi\left(t, t_{\mathrm{w}} ; H\right)$ in simulations that mimic the experimental field-cooling protocol (FC), where the temperature is lowered from above to below $T_{\mathrm{g}}$ in the presence of a constant magnetic field $H$.

We performed two independent simulations on Janus II at the critical temperature $T_{\mathrm{g}}=1.102(3)$ [27] (in IEA units) and at $T=1.05$ for several external magnetic fields and 16 samples. A protocol equivalent to FC, but convenient for simulations, is to place a random spin configuration instantaneously at the working temperature $T$, and turning on the external magnetic field at the same instant, so that $t_{\mathrm{w}}=0$. 
Spin-glass dynamics in the presence of a magnetic field: exploration of microscopic properties

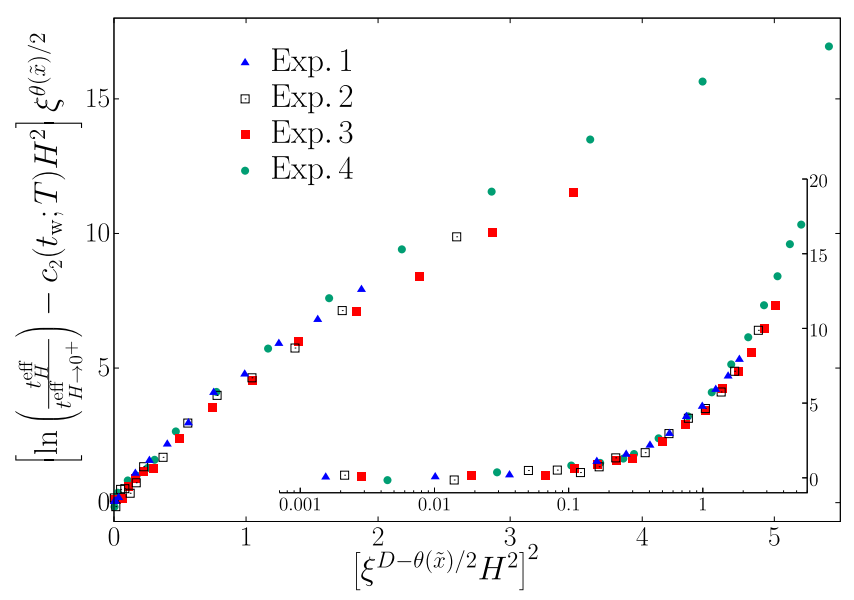

Figure 13. The non-linear parts from the experimental response time data, $\left[\ln t_{H}^{\text {eff }}-\right.$ $\left.c_{2}\left(t_{\mathrm{w}} ; T_{\mathrm{m}}\right) H^{2}\right] \xi^{\theta(\tilde{x}) / 2}$, plotted against $\left(\xi^{D-\theta(\tilde{x}) / 2} H^{2}\right)^{2}$. The deviations of the data at $T_{\mathrm{m}}=29 \mathrm{~K}$ may be caused by a shift in $T_{\mathrm{g}}$ as the temperature begins to approach $T_{\mathrm{g}}(H)$. The small- $x$ range is enlarged in the inset.

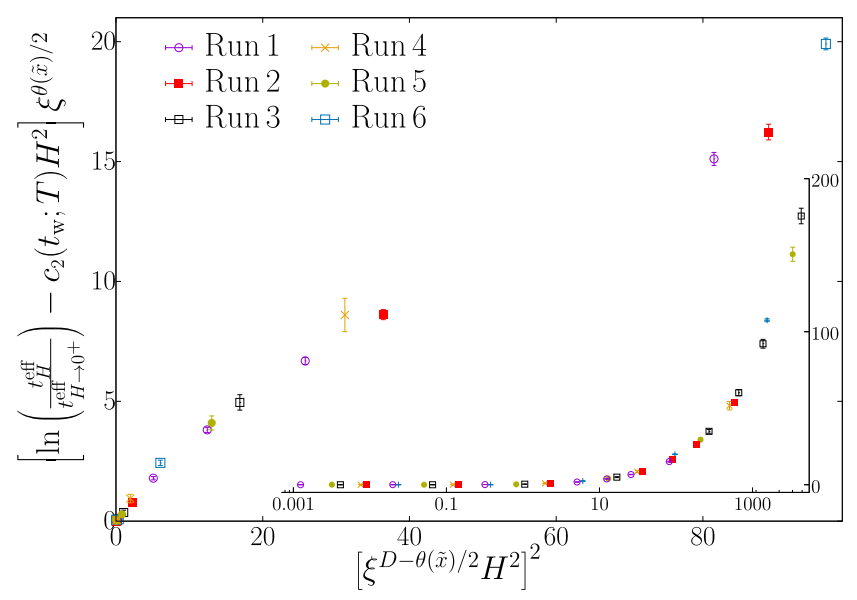

Figure 14. The non-linear parts from the numerical response time data, $\left[\ln \left(t_{H}^{\mathrm{eff}} / t_{H \rightarrow 0^{+}}^{\mathrm{eff}}\right)-c_{2}\left(t_{\mathrm{w}} ; T_{\mathrm{m}}\right) H^{2}\right] \xi^{\theta(\tilde{x} / 2)}$, plotted against $\left(\xi^{D-\theta(\tilde{x}) / 2} H^{2}\right)^{2}$. The abscissa of the main panel is in linear scale and shows a closeup for small values of $\left(\xi^{D-\theta(\tilde{x}) / 2} H^{2}\right)^{2}$. The abscissa of the insert is in log scale in order to report all our numerical data.

According to equation (20), at the critical temperature $T_{\mathrm{g}}$, and for small external magnetic fields $H$, there exists a scaling behaviour that connects $\xi\left(t, t_{\mathrm{w}} ; H\right)$ with the external magnetic field $H$ :

$$
\left[\xi\left(t, t_{\mathrm{w}} ; H\right) H^{2 / y_{H}}\right] \propto \text { const. }
$$

The correlation length $\xi\left(t, t_{\mathrm{w}} ; H\right)$ grows as

$$
\xi\left(t_{\mathrm{w}}\right) \propto t_{\mathrm{w}}^{1 / z(T)},
$$


Table 6. The aging-rate factors $z(T)$ used in figure 15 .

\begin{tabular}{ll}
\hline$T$ & $z(T)$ \\
\hline 1.1 & 6.60 \\
1.05 & 7.00 \\
1.0 & 7.30 \\
0.9 & 8.12 \\
\hline
\end{tabular}

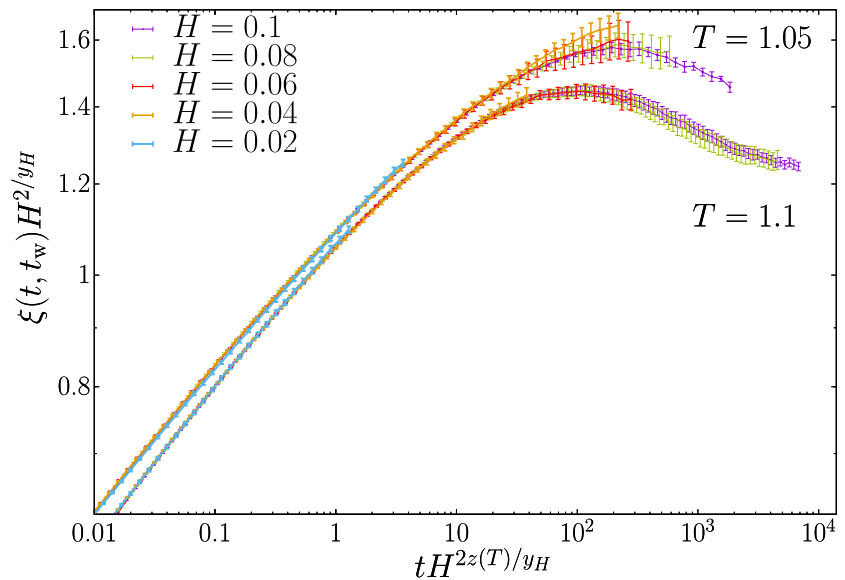

Figure 15. Critical dynamical scaling according to equations (54) and (57). We show data for $T=1.1 \approx T_{\mathrm{g}}[27]$ and for $T=1.05$.

with an exponent that, in a first approximation, is expected to behave near the critical temperature as [2]:

$$
z(T) \simeq z_{\mathrm{c}} \frac{T_{\mathrm{g}}}{T}, \quad \text { where } z_{\mathrm{c}}=z\left(T_{\mathrm{g}}\right)=6.69(6) .
$$

Hence, using equations (55) and (56) in the scaling argument of equation (54), we have equivalently,

$$
\left[t \times H^{2 z(T) / y_{H}}\right] \propto \text { const. }
$$

In table 6 we list the aging-rate factors $z(T)$ used in our analysis. We plot our rescaled data in figure 15 .

The agreement with the scaling prediction, evinced by the data collapse, is striking. Our plots also exhibit overshooting, as evidence for the paramagnetic phase when the magnetic field is turned on.

The reader could wonder why we have used equation (54) for the scaling analysis at the temperature $T=1.05<T_{\mathrm{g}}$, and whether this implies evidence of the absence of the dAT line in finite dimension. We shall address these questions in section 6 . 
Spin-glass dynamics in the presence of a magnetic field: exploration of microscopic properties

Table 7. Parameters of our ferromagnetic simulations for the Ising and Heisenberg models.

\begin{tabular}{lccc}
\hline System & $L$ & $H$ & Number of runs \\
\hline \multirow{3}{*}{ Ising } & & 0 & 2200 \\
& 256 & 0.001 & 2600 \\
& & 0.003 & 1700 \\
& & 0.005 & 1200 \\
\hline \multirow{3}{*}{ Heisenberg } & 200 & 0.003 & 2660 \\
& & 0.004 & 1000 \\
& & 0.005 & 2400 \\
\hline
\end{tabular}

5.6.2. Overshooting in a ferromagnetic system. By studying two ordered systems we can show that the overshooting phenomenon is, in fact, general. To demonstrate generality, we have simulated the three-dimensional Ising and Heisenberg models in a cubic lattice with periodic boundary conditions and size $L$ at the critical point $T_{\mathrm{c}}$. The $N=L^{D}$ $(D=3)$ Heisenberg spins interact with their lattice nearest neighbours through the Hamiltonian

$$
\mathcal{H}=-\sum_{\left\langle r, r^{\prime}\right\rangle} \boldsymbol{S}_{r} \cdot \boldsymbol{S}_{r^{\prime}}+\boldsymbol{H} \cdot \sum_{r} \boldsymbol{S}_{r}
$$

where $\boldsymbol{S}_{r}$ are unit vector spins and $\boldsymbol{H}$ is an external magnetic field. The connected correlation function is

$$
C(r, t)=\frac{1}{L^{3}} \sum_{x} \boldsymbol{S}_{x}(t) \cdot \boldsymbol{S}_{r+x}(t)-[\boldsymbol{m}(t)]^{2},
$$

with

$$
\boldsymbol{m}(t)=\frac{1}{L^{3}} \sum_{x} \boldsymbol{S}_{x}(t)
$$

We only write equations for the Heisenberg model, equations (58)-(60), but the Ising analogues can be obtained trivially by just dropping the vector symbol in the spins. Furthermore, the correlation function $C(r, t)$ will be averaged over different initial conditions (runs). We report the simulation details in table 7 .

The Ising and Heisenberg model have different symmetry properties, so they belong to two distinct universal classes. In other words, each model has a distinct value for the critical temperature and exponents. The Ising model has $\eta=0.0362978(20)$ [49], $z=2.0245(15)$ [50] and $\beta_{\mathrm{c}}=0.221654626(5)$ [51]. Instead, for the Heisenberg ferromagnet $\eta=0.378(3)[52,53], z=2.033(5)$ [54] and $\beta_{\mathrm{c}}=0.693001(10)$ [55] $\left(\beta_{\mathrm{c}} \equiv\right.$ $\left.1 / T_{\mathrm{c}}\right)$.

As explained in appendix A.5, the correlation length $\xi\left(t, t_{\mathrm{w}} ; H\right)$ can be calculated with integral estimators $[7,8]$,

$$
I_{k}\left(T, t_{\mathrm{w}}\right)=\int_{0}^{\infty} \mathrm{d} r r^{k} C\left(r, t_{\mathrm{w}} ; T\right), \quad \xi_{k, k+1}\left(t_{\mathrm{w}} ; T\right)=\frac{I_{k+1}\left(t_{\mathrm{w}} ; T\right)}{I_{k}\left(t_{\mathrm{w}} ; T\right)} .
$$


Spin-glass dynamics in the presence of a magnetic field: exploration of microscopic properties

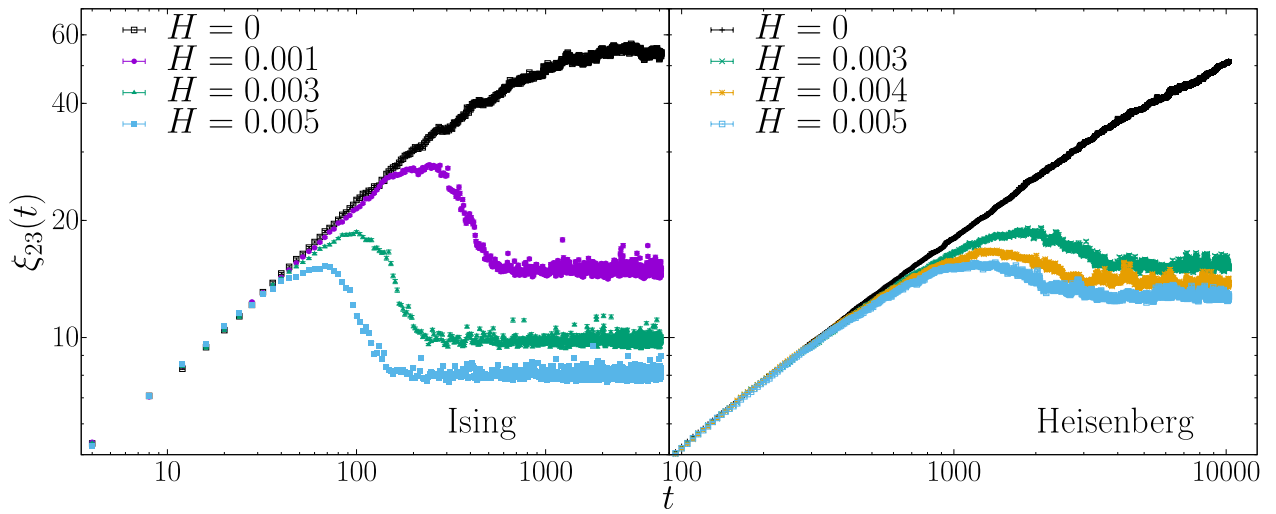

Figure 16. The $\log -\log$ plots show the behaviour of $\xi_{23}$ versus time for the Ising (left) and Heisenberg (right) models for three magnetic fields. All simulations were performed at the critical temperature $T_{\mathrm{c}}$ appropriate to each model. The saturation at long times exhibited by the Ising model at $H=0$ is a finite-size effect.

In this section, we evaluate the correlation length $\xi_{23}\left(t, t_{\mathrm{w}} ; H\right)$. As the reader can notice, the growth of $\xi_{23}(t)$ overshoots before reaching equilibrium for any external magnetic field for both ferromagnetic models, see figure 16 .

According to equation (20), at the critical temperature $T_{\mathrm{c}}$, and for small external magnetic fields $H$, there exists a scaling law that connects $\xi\left(t, t_{\mathrm{w}} ; H\right)$ with the external magnetic field $H$ in ferromagnetic system:

$$
\left[\xi\left(t, t_{\mathrm{w}} ; H\right) H^{1 / y_{H}}\right] \propto \text { const. }
$$

As the reader can notice, equation (62) differs from equation (54) in the power of the magnetic field. In the ferromagnetic system, the relevant external variable is $H$ and not $H^{2}$ as it would be for spin glasses [42, 43]. Analogously to equation (57), we can rescale time as

$$
\left[t \times H^{z(T) / y_{H}}\right] \propto \text { const } .
$$

We plot our rescaled data in figure 17. The agreement with the scaling prediction, both for the Heisenberg and for the Ising model, is remarkable.

In conclusion, the overshooting phenomenon is general and we have observed it both in ferromagnetic systems, figures 16 and 17, and in disordered ones, figure 15.

\section{Investigation of the dAT line in $D=3$}

The existence (or not) of the spin-glass condensation in the presence of a magnetic field remains the subject of some controversy (see, e.g. [56-59]). In a mean-field treatment, de Almeida and Thouless [60] showed that, for the Sherrington-Kirkpatrick infinite-range mean-field model [61], there would be a phase transition according to the following 
Spin-glass dynamics in the presence of a magnetic field: exploration of microscopic properties

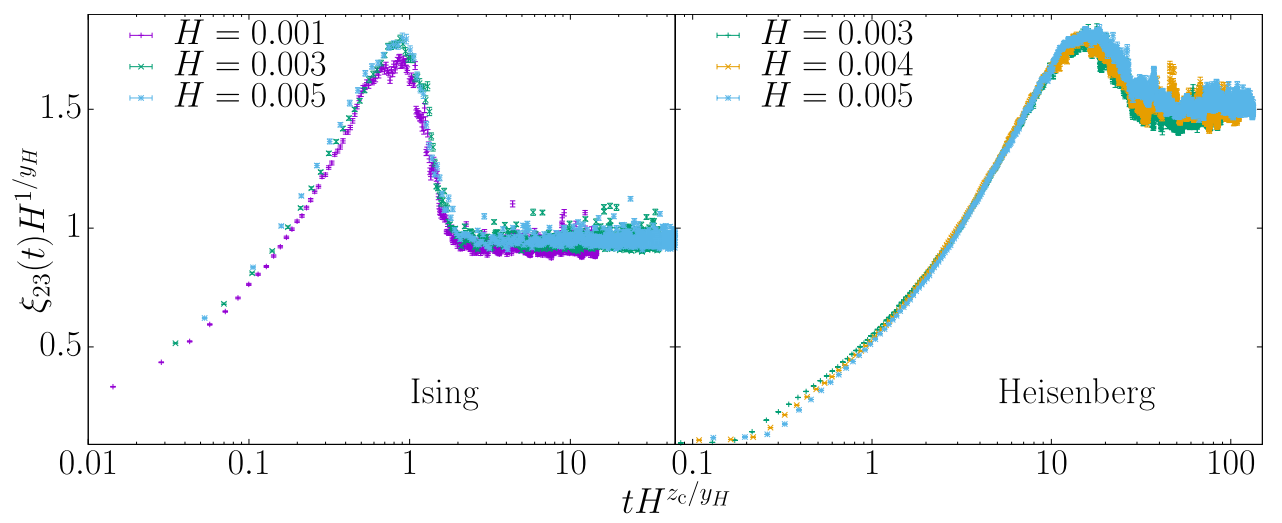

Figure 17. Critical dynamical scaling for ferromagnetic models. The data from figure 16 for the Ising (left) and Heisenberg (right) models for the three nonvanishing magnetic fields are rescaled following the predictions of the renormalisation group (see equations (62) and (63)). In this case the relevant variables are $\xi H^{1 / y_{H}}$ and $t H^{z_{c} / y_{H}}$, with $y_{H}=(D+2-\eta) / 2$ with $D=3$.

relationship for Ising spin glasses,

$$
\left(1-\frac{T_{\mathrm{g}}(H)}{T_{\mathrm{g}}(0)}\right)^{3}=\frac{3}{4} h^{2}
$$

with

$$
h=\frac{\mu H}{k_{\mathrm{B}} T_{\mathrm{g}}(0)},
$$

where $\mu$ is the spin magnetic moment. Conversely, the droplet model [62, 63] would predict no phase transition except exactly at $H=0$. This dispute was addressed by Lefloch et al [64]. Their final conclusion bears repetition: 'thus, even if the spin glass does not exist in a magnetic field, at least it looks the same as in zero field, as far as we experimentalists can see'.

In finite dimension and for $T$ very close to the critical temperature $T_{\mathrm{g}}(H=0)$, the dAT line, provided it exists, should be governed by the Fisher-Sompolinsky [31] relation:

$$
\left(1-\frac{T_{\mathrm{g}}(H)}{T_{\mathrm{g}}(0)}\right) \propto H^{4 / \nu(5-\eta)}
$$

where we have specialised to $D=3 \cdot{ }^{23}$ Rather than through $T_{\mathrm{g}}(H)$, we are interested in describing the dAT line geometrically by the inverse function of $T_{\mathrm{g}}(H)$, namely $H_{\mathrm{c}}(T)$. Hence, we rewrite equations (64) and (66) as

$$
\text { Mean - field: } H_{\mathrm{c}}(T) \propto\left(1-\frac{T}{T_{\mathrm{g}}}\right)^{a_{\mathrm{MF}}}, \quad a_{\mathrm{MF}}=1.5,
$$

\footnotetext{
${ }^{23}$ Notice this is the same relation used for matching the numerical and experimental scales in section 3.
} 
Spin-glass dynamics in the presence of a magnetic field: exploration of microscopic properties

$$
3 \mathrm{D}: H_{\mathrm{c}}(T) \propto\left(1-\frac{T}{T_{\mathrm{g}}}\right)^{a_{3 \mathrm{D}}}, \quad a_{3 \mathrm{D}}=\frac{\nu(5-\eta)}{4} \quad \rightarrow \quad a_{3 \mathrm{D}}=3.45(5),
$$

where we have taken the 3D critical exponents $\nu$ and $\eta$ from [27]. The following considerations, based on equations (67) and (68), will be useful:

- $H_{\mathrm{c}}(T)$ is a decreasing function of $T$ (remember $T \leqslant T_{\mathrm{g}}$ ) and $H_{\mathrm{c}}\left(T_{\mathrm{g}}\right)=0$. This means that, upon approaching $T_{\mathrm{g}}$ from below, one eventually crosses the dAT line for any $H>0$, no matter how small $H$ is.

- When $H>H_{\mathrm{c}}(T)$ the system is above the dAT line, in its paramagnetic phase: the correlation length, $\xi\left(t, t_{\mathrm{w}} ; H\right)$, reaches asymptotically its equilibrium value $\xi_{\text {eq }}(H)$ for very long time $t$.

- When $H<H_{\mathrm{c}}(T)$ we are in the spin-glass phase and one expects to observe a powerlaw growth of the correlation length, see equation (55).

- The $a_{3 \mathrm{D}}$ exponent is much larger than the mean-field $(\mathrm{MF})$ one, $a_{3 \mathrm{D}} \approx 2.3 \times a_{\mathrm{MF}}$. This implies that, in $D=3$, the dAT line is very flat when one approaches the critical temperature $T \simeq T_{\mathrm{g}}$.

In particular, our last item above suggests an interpretation of the somewhat surprizing results in figure 15 , where data for $T=1.05$ were successfully scaled with the scaling law appropriate for $T_{\mathrm{g}}$ (recall that $1.05<T_{\mathrm{g}}=1.102(3)$ and that at $T=T_{\mathrm{g}}$ we are in the paramagnetic phase for any $H>0$ ). Assuming that the proportionality coefficient is of order unity, let us estimate the critical magnetic field at $T=1.05$ exploiting equation (68):

$$
H_{\mathrm{c}}(T=1.05) \sim 3 \times 10^{-5} .
$$

Considering, now, that the smallest magnetic field in figure 15 , namely $H=0.02$, is larger than $H_{\mathrm{c}}(T=1.05)$ by a factor of 1000 or so, there is little surprize in that a scaling law assuming $H_{\mathrm{c}}(T=1.05)=0$ works with our data.

Our focus in this section will be an exploration of the growth of the spin-glass correlation length, $\xi\left(t, t_{\mathrm{w}} ; H\right)$, under conditions that mimic the experimental protocol for measurement of the zero-field-cooled magnetisation, $M_{\mathrm{ZFC}}\left(t, t_{\mathrm{w}} ; H\right)$ for $t_{\mathrm{w}} \neq 0$, recall section 3 .

In figure 18 we plot $\xi\left(t, t_{\mathrm{w}} \neq 0 ; H\right)$ as a function of time for different magnetic fields $H$. We compute $\xi$ from the microscopic correlation function $C_{4}(r)$ (see appendix A.5), which requires that we compute error bars from the sample-to-sample fluctuations. We have simulated different samples only for some values of $H$ and $t_{\mathrm{w}}$ because of the enormous computational effort involved. We show error bars in figure 18 only in those cases where they can be computed.

The time evolution of the spin-glass correlation length $\xi\left(t, t_{\mathrm{w}} ; H\right)$ depends markedly on the interplay between the waiting time $t_{\mathrm{w}}$ and the value of the magnetic field $H$, see figure 18. The system needs several time steps before responding to the switching on of the magnetic field. Different scenarios appear.

On the one hand, for the largest magnetic fields, namely $H>0.04$ both at $T=0.9$ and $T=1.0$, the correlation length displays a non-monotonic time behaviour, just as we found in section 5.6.1 for the dynamics in the paramagnetic phase (recall 
Spin-glass dynamics in the presence of a magnetic field: exploration of microscopic properties

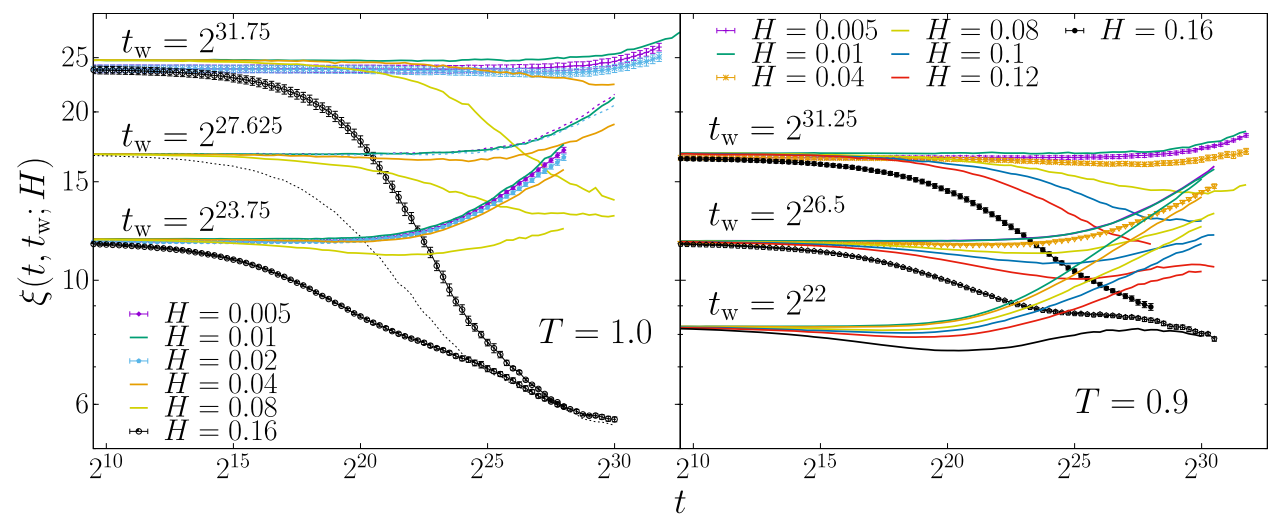

Figure 18. Growth of $\xi\left(t, t_{\mathrm{w}} \neq 0 ; H\right)$ in simulations that mimic the experimental ZFC protocol. Plots are in log-log scale.

that $t_{\mathrm{w}}=0$ in section 5.6.1). In particular, for those cases when the starting correlation length, $\xi\left(t=0, t_{\mathrm{w}} ; H\right)$, is larger than the equilibrium value $\xi_{\text {eq }}(H)$, the correlation length decays. Otherwise, we observe an overshooting phenomenon reminiscent of our findings in section 5.6.1, see figure 18.

On the other hand, for $H<0.08$, we observe that the correlation length $\xi\left(t, t_{\mathrm{w}} ; H\right)$ appears to follow the same power-law growth for all the different waiting times. Here we must distinguish between the mean-field $H_{\mathrm{c}}^{\mathrm{MF}}(T)$ and the Fisher-Sompolinsky scaling $H_{\mathrm{c}}^{3 \mathrm{D}}(T)$, i.e. between equations (67) and (68). Using equation (64) for the former, one finds

$$
H_{\mathrm{c}}^{\mathrm{MF}}(T=0.9) \approx 0.0675 \text { and } H_{\mathrm{c}}^{\mathrm{MF}}(T=1.0) \approx 0.02421
$$

Interestingly, the scaling result, equation (68), yields

$$
H_{\mathrm{c}}^{3 \mathrm{D}}(T=0.9) \approx 0.003 \text { and } H_{\mathrm{c}}^{3 \mathrm{D}}(T=1.0) \approx 0.0003 .
$$

For the magnetic fields used in our simulations, therefore, one is presumably in the condensed state for $0.005 \leqslant H<0.08$ from the perspective of the MF solution of the Sherrington-Kirkpatrick model [61], while from the perspective of the Fisher-Sompolinsky scaling [31] one is always in the paramagnetic state since $H>H_{\mathrm{c}}^{3 \mathrm{D}}(T=0.9,1.0)$. Though this latter region is not accessible experimentally through magnetic measurements, one can argue that the simulation results should be symmetric around $T_{\mathrm{c}}(H)$. This is the basis for the comparison between experiment and simulations contained in section 5 of this paper.

Let us now attempt a scaling analysis similar to the one in section 5.6.1 for those magnetic field values for which the asymptotic $\xi_{\text {eq }}(H)$ can be at least guessed from figure 18. We start by modifying scaling relationship (54) to

$$
\xi\left(t, t_{\mathrm{w}} ; H\right)\left|H^{2}-H_{\mathrm{c}}^{2}(T)\right|^{1 / y_{H}} \propto \text { const. }
$$

Next, using equation (55) in the scaling argument of equation (72), we have, equivalently,

$$
\left(t \times\left|H^{2}-H_{\mathrm{c}}^{2}(T)\right|^{z(T) / y_{H}}\right) \propto \text { const. }
$$


Spin-glass dynamics in the presence of a magnetic field: exploration of microscopic properties

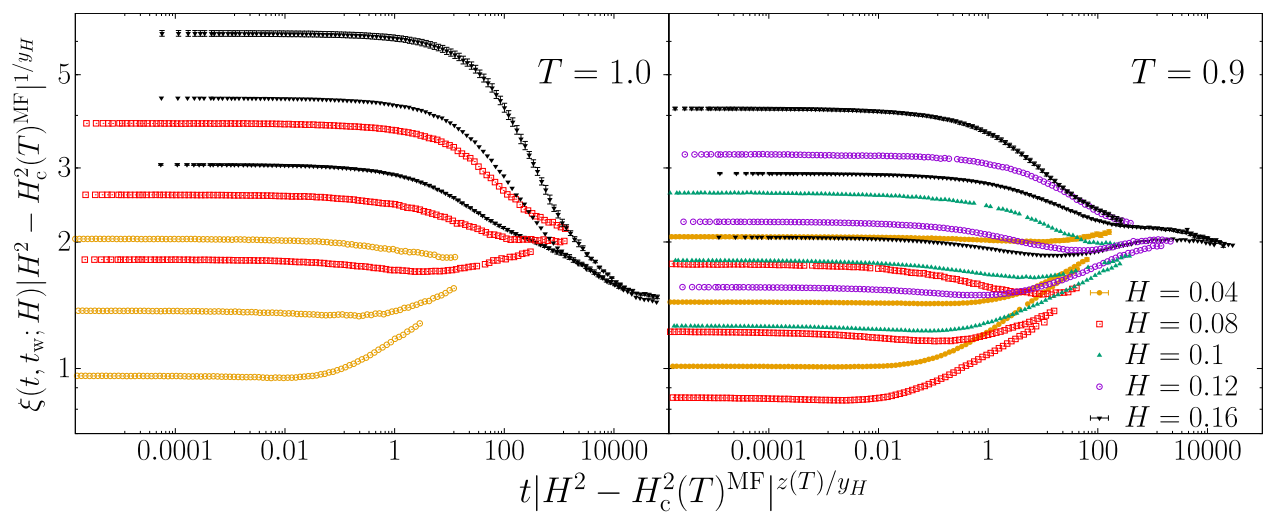

Figure 19. Search for the dAT line in $D=3$ using MF scaling. Plots are in log-log scale and show the behaviour of the rescaled quantities defined in equations (72) and (73) for the mean-field estimators $H_{\mathrm{c}}(T)^{\mathrm{MF}}$, see equation (70). The ageing rates $z(T)$ used in this scaling are listed in table 6, to be found in section 5.6.

We replot our rescaled data in figure 19 for the mean-field values of $H_{\mathrm{c}}^{\mathrm{MF}}(T)$, see equation (70). As seen in the panel for $T=0.9$, there is nearly perfect scaling for $H \geqslant 0.08$ but not for $H=0.04$, though the curves do seem to coalesce for the three different waiting times.

It is tempting to suggest that, for this value of magnetic field, one is in the condensed phase. Glancing at figure 18, however, the growth of $\xi\left(t, t_{\mathrm{w}} ; H\right)$ for $H=0.04$ breaks away from the curves for the larger magnetic fields, so that it is very possible that it would join the equilibrium curves (i.e. the paramagnetic regime) at times longer than those accessible in our simulations. This ambiguity softens the interpretation that we have broached the dAT line in our simulations, as would be predicted from a MF approach.

However, if we replot our data using the scaling result of equation (71), as exhibited in figure 20, for the values of $H_{\mathrm{c}}^{3 \mathrm{D}}(T)$ for $T=0.9$, the data appear to collapse for all of the magnetic fields, including $H=0.04\left[H_{\mathrm{c}}^{3 \mathrm{D}}(T=0.9) \ll 0.04\right]$. The Fisher-Sompolinsky scaling would, therefore, support the conjecture that at $T=0.9$, our simulation results for $H=0.04$ are in the paramagnetic regime.

As to the rescaled $T=1.0$ data in figures 19 and 20, they are of low quality, limiting the magnetic fields to relatively large values. The three values $(H=0.04,0.08,0.16)$ for which it is feasible to rescale are all above the $H_{\mathrm{c}}^{\mathrm{MF}, 3 \mathrm{D}}(T=1.0)$ values given by equations (70) and (71). All are, hence, in the paramagnetic regime, as can seen from the shape of the curves in both figures.

Thus, though the data of figure 18 suggests that, for the lowest magnetic fields and $T=0.9, \xi\left(t, t_{\mathrm{w}} ; T\right)$ may be growing as a power law, and thus be in the condensed phase, our limited time scale for the simulations is unable to conclude that we have, in fact, straddled the dAT line. If one assumes Fisher-Sompolinsky scaling, equation (71), all of our simulation results would be in the paramagnetic region. Until much longer times scales become reachable (either at lower temperatures, or smaller magnetic fields), even our powerful Janus II simulations are unable to arrive at a definitive conclusion regarding the existence, or non-existence, of the dAT line for $D=3$ Ising spin glasses. 
Spin-glass dynamics in the presence of a magnetic field: exploration of microscopic properties

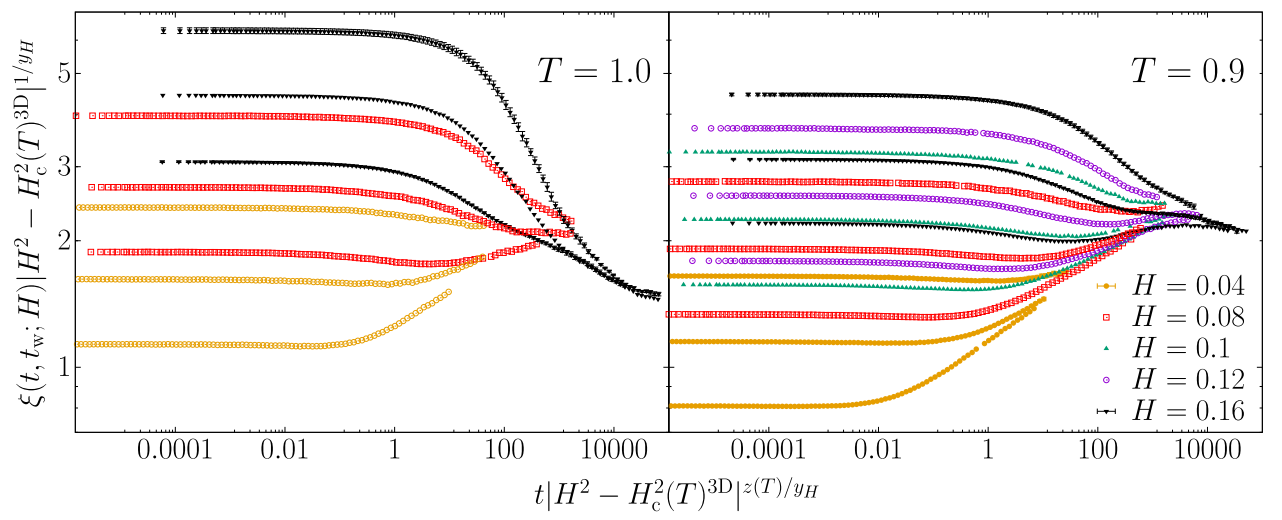

Figure 20. Search for the dAT line in $D=3$ with Fisher-Sompolinsky scaling. Plots are in $\log -\log$ scale and show the behaviour of the rescaled quantities defined in equations (72) and (73) for the Fisher-Sompolinsky estimators $H_{\mathrm{c}}(T)^{3 \mathrm{D}}$, see equation (71). We report the ageing rates $z(T)$ used in this scaling in table 6 to be found in section 5.6.

\section{Conclusions}

This paper demonstrates the unique and powerful combination of experiment, theory, and simulations addressing complex dynamics. The use of single crystals enables experiments to exhibit the consequences of very large spin-glass correlation lengths. The power of a special-purpose computer, Janus II, in combination with theory, is sufficient to extend simulation time and length scales to values explored experimentally. Together, these approaches unite to develop new and important insights into spin-glass dynamics.

Previous work [5, 23] explored the reduction of the free-energy barrier heights responsible for ageing in spin glasses by the Zeeman (magnetic field $H$ ) energy. Observations for small magnetic fields, proportional to $H^{2}$, were used to extract a quantitative value for the spin-glass correlation length and its growth rate with time. As the magnetic field was increased, however, departures from proportionality to $H^{2}$ were observed. This paper presents detailed experimental observations of this behaviour and, together with theory [4], is able to demonstrate the applicability of a new non-linear scaling law for the magnetisation in the vicinity of the spin-glass condensation temperature $T_{\mathrm{g}}$. Remarkably, Janus II simulations were able to generate comparable values for the magnetisation dynamics, with the added value of direct measurement of the characteristic response time.

The combination of these two approaches has put to rest a decades-old controversy concerning the nature of the Zeeman energy. We have shown that the departures from proportionality to $H^{2}$ are caused by non-linear terms in the magnetisation, and not by fluctuations of the magnetisation that lead to a Zeeman energy proportional to $H$. Further, the departure from an $H^{2}$ behaviour that was used to justify the proportionality to $H$ is shown to be a consequence of non-linear behaviour of the magnetisation in $H$, and fully accounted for using the new scaling law. This is an important finding, because otherwise the extraction of the spin-glass correlation length from the Zeeman-energy reduction in the barrier height would have been in error. 
One of the most interesting findings in the paper is the extraction of the characteristic response time for spin glasses, $t_{H}^{\text {eff }}$, from simulations. It has been made possible by noting that the spin-glass correlation function reaches a peak at the response time. That is,

$$
C\left(t_{H}^{\mathrm{eff}}, t_{\mathrm{w}} ; H\right)=C_{\text {peak }}\left(t_{\mathrm{w}}\right) .
$$

Thus, by extracting $C_{\text {peak }}\left(t_{\mathrm{w}}\right)$ one can determine the characteristic response time $t_{H}^{\text {eff }}$. It is this observation that enables simulations to give quantitative values for the non-linear magnetic susceptibility that can be compared with the new scaling law.

In addition, we have explored the microscopic behaviour of the magnetic states through the growth of the correlation lengths under two experimental protocols: ZFC and FC. We have proved that in a system close enough to the condensation temperature $T_{\mathrm{g}}$, the Fisher-Sompolinsky scaling relation holds under out-of-equilibrium conditions (see section 5.5). This will enable us in future simulations to compare the magnitude and growth of the spin-glass correlation length under two experimental protocols: the dynamics of zero-field-cooled and thermo-remanent magnetisations. The important point here is that this paper shows that our analysis will be valid under these non-equilibrium conditions.

We have discovered an overshooting phenomenon that is shown to be general for both ordered and disordered magnetic systems. And finally, we have explored the nature of the spin-glass condensation at $T_{\mathrm{g}}$ as a function of the external magnetic field, the socalled dAT line. We have presented preliminary evidence for its existence as a true condensation transition, but this conclusion should be regarded as provisional.

In conclusion, this paper has explored the nature of the spin-glass state in the vicinity of its condensation temperature $T_{\mathrm{g}}$. We displayed the power of combining insights from both experiment and simulations, coupled together by theory. We look forward to continued investigation of spin-glass dynamics using this relationship as we examine the microscopic nature of such phenomena as rejuvenation and memory. Finally, because spin-glass dynamics has applications in many diverse fields (ecology, biology, optimisation, and even social science), our work demonstrates that modelling complex systems is feasible in finite dimensions.

\section{Acknowledgments}

We are grateful for helpful discussions with S Swinnea about sample characterisation. This work was partially supported by the US Department of Energy, Office of Basic Energy Sciences, Division of Materials Science and Engineering, under Award No. DE-SC0013599, and Contract No. DE-AC02-07CH11358. We were partly funded as well by Ministerio de Economía, Industria y Competitividad (MINECO, Spain), Agencia Estatal de Investigación (AEI, Spain), and Fondo Europeo de Desarrollo Regional (FEDER, EU) through Grants No. FIS2016-76359-P, No. PID2019-103939RB-I00, No. PGC2018-094684-B-C21 and PGC2018-094684-B-C22, by the Junta de Extremadura (Spain) and Fondo Europeo de Desarrollo Regional (FEDER, EU) through Grant Nos. GRU18079 and IB15013 and by the DGA-FSE (Diputación General de Aragón-Fondo Social Europeo). This project has also received funding from the European Research 
Council (ERC) under the European Union's Horizon 2020 research and innovation programme (Grant No. 694925 LotGlasSy). D Y was supported by the Chan Zuckerberg Biohub and IGAP was supported by the Ministerio de Ciencia, Innovación y Universidades (MCIU, Spain) through FPU Grant No. FPU18/02665. B S was supported by the Comunidad de Madrid and the Complutense University of Madrid (Spain) through the Atracción de Talento programme (reference 2019-T1/TIC-12776). The simulations of section 5.6.2 were carried out at the ICCAEx supercomputer centre in Badajoz. We thank the staff at ICCAEx for their assistance.

\section{Appendix A. Technical details about our simulations}

\section{A.1. Smoothing and interpolating the data}

Our numerical data for the magnetisation at small magnetic fields are rather noisy, which complicates the process of taking its derivative with respect to $\ln t$. This derivative is the response function $S\left(t, t_{\mathrm{w}} ; H\right)$ (recall equation (11)). This is why, before differentiating, we have followed a de-noising method first proposed in [45]. Because we work at larger correlation lengths (and closer to $T_{\mathrm{g}}$ ) than in that work, however, we have found it preferable to change some technical details. We explain below the precise de-noising method that we have followed in this work.

Our starting observation is that the derivative of both $M_{\mathrm{ZFC}}\left(t, t_{\mathrm{w}} ; H\right)$ and $T M_{\mathrm{ZFC}}\left(t, t_{\mathrm{w}} ; H\right) / H$ peak at exactly the same time $t_{H}^{\mathrm{eff}}$. However, $T M_{\mathrm{ZFC}}\left(t, t_{\mathrm{w}} ; H\right) / H$ enjoys the advantage of being a very smooth function of the correlation $C\left(t, t_{\mathrm{w}} ; H\right)$. This smooth function is named the FDR [32-36]. The key point is that, at variance with the magnetisation, $C\left(t, t_{\mathrm{w}} ; H\right)$ can be computed with high accuracy for any value of the field $H$, including $H=0$. Thus, we follow a simple two-step de-noising algorithm:

(a) We fit our data for $T M_{\mathrm{ZFC}}\left(t, t_{\mathrm{w}} ; H\right) / H$ as a function of $C\left(t, t_{\mathrm{w}} ; H\right)$, see equation (A.1).

(b) We replace our data for $T M_{\mathrm{ZFC}}\left(t, t_{\mathrm{w}} ; H\right) / H$ by the just-mentioned fitted function evaluated at $C\left(t, t_{\mathrm{w}} ; H\right)$.

Our chosen functional form is as follows. Let the quantity $T M_{\mathrm{ZFC}}\left(t, t_{\mathrm{w}} ; H\right) / H$ be approximated by $f(\hat{x})$, where for notational simplicity we do not write the explicit dependence on $t, t_{\mathrm{w}}$ and $H$,

$$
f(\hat{x})=f_{\mathrm{L}}(\hat{x}) \frac{1+\tanh [\mathcal{Q}(\hat{x})]}{2}+f_{\mathrm{s}}(\hat{x}) \frac{1-\tanh [\mathcal{Q}(\hat{x})]}{2}
$$

with $\mathcal{Q}(\hat{x})=\left(\hat{x}-x^{*}\right) / w$. The function $f(\hat{x})$ has distinct behaviour for large and small $\hat{x}$. The crossover between the two functional forms is smoothed by the $\tanh [\mathcal{Q}(\hat{x})]$ functional term, where $x^{*}$ is the crossover point and $w$ is the crossover rate. The functional form for small $\hat{x}$ is

$$
f_{\mathrm{s}}(\hat{x})=a_{0}+\sum_{k=1}^{N} a_{k} \frac{\left(\hat{x}-\hat{x}_{\min }\right)^{k}}{k !} .
$$


Spin-glass dynamics in the presence of a magnetic field: exploration of microscopic properties

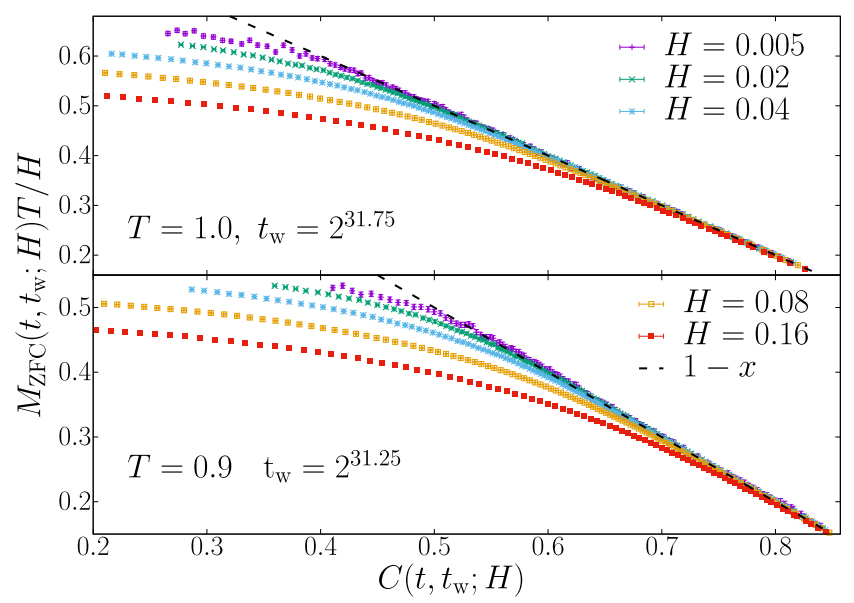

Figure 21. The behaviour of $T M_{\mathrm{ZFC}}\left(t, t_{\mathrm{w}} ; H\right) / H$ is exhibited as a function of $C\left(t, t_{\mathrm{w}} ; H\right)$. (Top) $T=1.0, t_{\mathrm{w}}=2^{31.75}$. (Bottom) $T=0.9, t_{\mathrm{w}}=2^{31.25}$. We do not report all the magnetic values for simplicity.

For the large- $\hat{x}$ region, we choose a polynomial expansion in terms of $(1-\hat{x})$ :

$$
f_{\mathrm{L}}(\hat{x})=(1-\hat{x})+\sum_{k=2}^{N^{\prime}+1} b_{k} \frac{(1-\hat{x})^{k}}{k !} .
$$

The polynomial expansion in $1-\hat{x}$ is quite natural in the large- $\hat{x}$ region [45], as a deviation from the fluctuation-dissipation theorem. This theorem, which holds only under equilibrium conditions, predicts $N^{\prime}=0$ for equation (A.3) and $x^{*}=w=0$ for equation (A.1) (so that, in equilibrium, one would have $f(\hat{x})=(1-\hat{x})$ in equation (A.1)). In the small- $\hat{x}$ region, there is not a strong justification (other than convenience) for our choice of $f_{\mathrm{s}}(\hat{x})$. In fact, the choice of [45] for $f_{\mathrm{s}}(\hat{x})$ was a Padé approximant. The quantity $T M_{\mathrm{ZFC}}\left(t, t_{\mathrm{w}} ; H\right) / H$ turns out to be affected by strong non-linear effects that increase with increasing external magnetic field $H$ and upon approaching the glass temperature $T_{\mathrm{g}}$ (see figure 21 ).

As we discuss in appendix A.2, it is necessary to select the appropriate order for the polynomials in equations (A.2) and (A.3). Our preferred choices are given in table 8.

Errors are computed using a jackknife procedure. We perform an independent fit for each jackknife block, and compute errors from the jackknife fluctuations of the fitted $f(\hat{x})$. In figure 22 we show a comparison between the original and smoothed data for the case $T=1.0$ and $t_{\mathrm{w}}=2^{31.75}$. As expected, the de-noising technique is most important for the smallest magnetic fields.

\section{A.2. Over-fitting problem}

A difficulty in our fits to equation (A.1) is that we can use only the diagonal part of the covariance matrix in the computation of the goodness-of-fit indicator $\chi^{2}$. This is the reason underlying the very small values for $\chi^{2}$ that we show in table 8 . As a consequence, we cannot trust the $\chi^{2}$ test for selecting the appropriate order for the 
Spin-glass dynamics in the presence of a magnetic field: exploration of microscopic properties

Table 8. For each of our fits to equation (A.1) we report: the order of the polynomial in equation (A.2) $N$, the number of fitted parameters in equation (A.3) $N^{\prime},\left[N^{\prime}=0\right.$ means $f_{L}(\hat{x})=$ $(1-\hat{x})]$, the crossover parameters $x^{*}$ and $w$, and the fit's figure of merit $\chi^{2} /$ d.o.f. (d.o.f. stands for degrees of freedom). Note that we can only compute the so-called diagonal $\chi^{2}$, which takes into account only the diagonal elements of the covariance matrix. Because of this limitation, we find a value of $\chi^{2}$ significantly smaller than the number of degrees of freedom for many of our fits.

\begin{tabular}{|c|c|c|c|c|c|c|c|}
\hline & $t_{\mathrm{w}}$ & $H$ & $N$ & $N^{\prime}$ & $x^{*}$ & $w$ & $\chi^{2} /$ d.o.f. \\
\hline \multirow{23}{*}{$T=0.9$} & \multirow{7}{*}{$2^{22}$} & 0.010 & 1 & 1 & $0.583(11)$ & $0.128(1)$ & $45.721 / 110$ \\
\hline & & 0.020 & 1 & 1 & $0.604(6)$ & $0.1264(6)$ & $49.874 / 110$ \\
\hline & & 0.040 & 3 & 2 & $0.589(4)$ & $0.099(4)$ & $27.991 / 107$ \\
\hline & & 0.080 & 3 & 2 & $0.576(7)$ & $0.164(11)$ & $64.344 / 107$ \\
\hline & & 0.100 & 3 & 2 & $0.681(12)$ & $0.187(4)$ & $33.133 / 99$ \\
\hline & & 0.120 & 3 & 3 & $0.665(18)$ & $0.0725(5)$ & $31.709 / 98$ \\
\hline & & 0.160 & 4 & 4 & $0.62(4)$ & $0.052(1)$ & $65.326 / 104$ \\
\hline & \multirow{8}{*}{$2^{26.5}$} & 0.005 & 2 & 0 & $0.530(17)$ & $0.100(7)$ & $28.518 / 112$ \\
\hline & & 0.010 & 1 & 1 & $0.520(10)$ & $0.115(4)$ & $37.343 / 112$ \\
\hline & & 0.020 & 2 & 0 & $0.535(3)$ & $0.091(3)$ & $35.535 / 112$ \\
\hline & & 0.040 & 2 & 0 & $0.653(2)$ & $0.033(2)$ & $59.325 / 112$ \\
\hline & & 0.080 & 3 & 2 & $0.585(7)$ & $0.161(11)$ & $55.006 / 109$ \\
\hline & & 0.100 & 3 & 2 & $0.674(12)$ & $0.176(4)$ & $32.874 / 103$ \\
\hline & & 0.120 & 3 & 3 & $0.68(6)$ & $0.078(11)$ & $42.782 / 102$ \\
\hline & & 0.160 & 4 & 4 & $0.623(15)$ & $0.034(8)$ & $77.297 / 106$ \\
\hline & \multirow{8}{*}{$2^{31.25}$} & 0.005 & 1 & 0 & $0.503(8)$ & $0.074(7)$ & $35.145 / 123$ \\
\hline & & 0.010 & 1 & 1 & $0.520(12)$ & $0.139(2)$ & $32.331 / 118$ \\
\hline & & 0.020 & 1 & 2 & $0.550(7)$ & $0.0335(3)$ & $31.219 / 121$ \\
\hline & & 0.040 & 2 & 0 & $0.554(16)$ & $0.080(2)$ & $75.790 / 116$ \\
\hline & & 0.080 & 3 & 2 & $0.598(6)$ & $0.152(8)$ & $38.439 / 115$ \\
\hline & & 0.100 & 3 & 2 & $0.688(10)$ & $0.170(4)$ & $29.904 / 107$ \\
\hline & & 0.120 & 3 & 3 & $0.655(14)$ & $0.062(4)$ & $32.396 / 98$ \\
\hline & & 0.160 & 4 & 4 & $0.549(22)$ & $0.077(6)$ & $67.296 / 112$ \\
\hline \multirow{11}{*}{$T=1.0$} & \multirow{6}{*}{$2^{23.75}$} & 0.005 & 1 & 0 & $0.371(18)$ & $0.178(12)$ & $60.709 / 103$ \\
\hline & & 0.010 & 1 & 0 & $0.411(4)$ & $0.129(4)$ & $46.709 / 103$ \\
\hline & & 0.020 & 1 & 0 & $0.397(2)$ & $0.138(2)$ & $76.009 / 103$ \\
\hline & & 0.040 & 2 & 0 & $0.457(2)$ & $0.146(2)$ & $49.401 / 102$ \\
\hline & & 0.080 & 4 & 0 & $0.589(6)$ & $0.066(1)$ & $36.384 / 100$ \\
\hline & & 0.160 & 4 & 3 & $0.639(12)$ & $0.061(6)$ & $48.338 / 98$ \\
\hline & \multirow{5}{*}{$2^{27.625}$} & 0.005 & 1 & 0 & $0.37(2)$ & $0.157(1)$ & $34.6242 / 101$ \\
\hline & & 0.010 & 1 & 0 & $0.400(8)$ & $0.128(5)$ & $45.169 / 111$ \\
\hline & & 0.020 & 1 & 0 & $0.389(2)$ & $0.132(2)$ & $53.121 / 111$ \\
\hline & & 0.040 & 3 & 0 & $0.559(11)$ & $0.049(4)$ & $32.738 / 109$ \\
\hline & & 0.080 & 3 & 1 & $0.638(8)$ & $0.023(11)$ & $491.701 / 108$ \\
\hline
\end{tabular}


Table 8. continued

\begin{tabular}{lllllll}
\hline & 0.160 & 3 & 1 & $0.667(2)$ & $0.023(3)$ & $140.853 / 108$ \\
\hline & 0.005 & 2 & 0 & $0.357(9)$ & $0.170(12)$ & $41.854 / 127$ \\
$2^{31.75}$ & 0.010 & 1 & 0 & $0.114(10)$ & $0.114(10)$ & $39.202 / 123$ \\
& 0.020 & 2 & 0 & $0.488(8)$ & $0.116(7)$ & $40.968 / 118$ \\
& 0.040 & 3 & 0 & $0.534(12)$ & $0.070(4)$ & $33.579 / 109$ \\
& 0.080 & 3 & 1 & $0.631(4)$ & $0.023(5)$ & $271.914 / 108$ \\
& 0.160 & 4 & 0 & $0.686(9)$ & $0.080(3)$ & $160.490 / 108$ \\
\hline
\end{tabular}

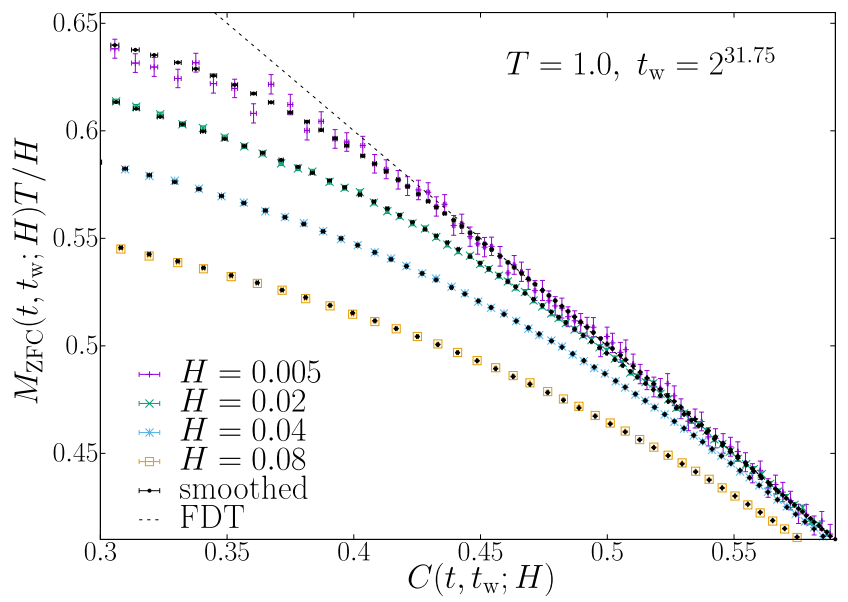

Figure 22. Comparison between the original and smoothed data at $T=1.0$ and waiting time $t_{\mathrm{w}}=2^{31.75}$. One can clearly see the advantage of the de-noising method for the lowest magnetic field.

polynomial expansions in equations (A.2) and (A.3). Hence, we follow a different strategy.

Fortunately, we can also compare the statistical errors that we find for the de-noised $T M_{\mathrm{ZFC}}\left(t, t_{\mathrm{w}} ; H\right) / H$ with different choices of the polynomial expansion (remember that these errors are not computed from $\chi^{2}$, but from the jackknife fluctuations). As an example, consider the case at $T=0.9$ for a waiting time $t_{\mathrm{w}}=2^{31.75}$ and $H=0.002$, which is exhibited in figure 23. The figure compares the statistical errors of the original, non-de-noised data with the errors found with two possible choices for the polynomial fits in equations (A.2) and (A.3). Although both fits are indistinguishable from the point of view of the $\chi^{2}$ test, see table 9 , the resulting errors are very different. In one case, we find statistical errors that evolve rather smoothly with $t$. For the second choice, we find wild oscillations in the size of the errors as $t$ varies. When in doubt, we have always taken the choice that provides the smoother $t$ evolution for the errors. As we said above, our final choices are reported in table 8 . 
Spin-glass dynamics in the presence of a magnetic field: exploration of microscopic properties

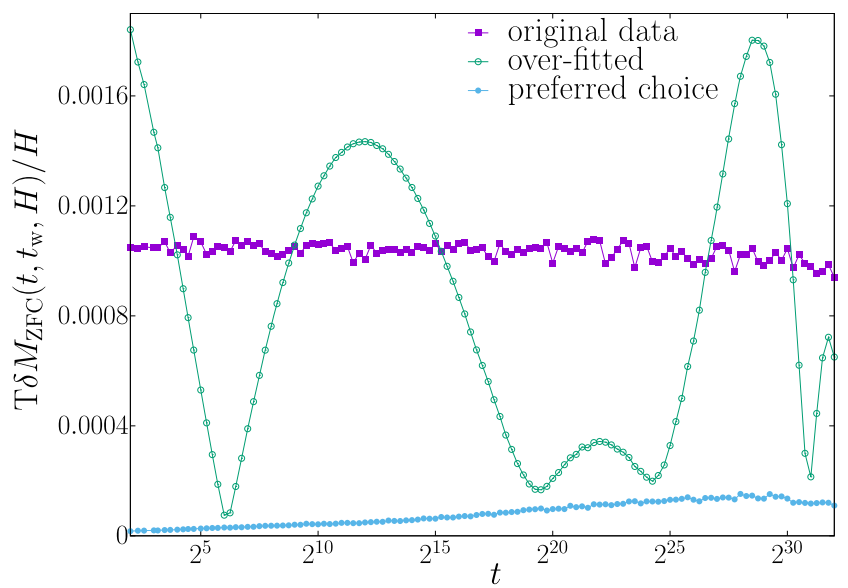

Figure 23. Comparison between the errors for the original and the de-noised data for $T M_{\mathrm{ZFC}}\left(t, t_{\mathrm{w}} ; H\right) / H$ (with $T=0.9, t_{\mathrm{w}}=2^{31.75}$ and $H=0.002$ ), for the two different fitting functions $f(\hat{x})$ reported in table 9 .

Table 9. Details about the two fits shown in figure 23. We follow the same notational conventions of table 8 .

\begin{tabular}{lccccc}
\hline Case & $N$ & $N^{\prime}$ & $x^{*}$ & $w$ & $\chi^{2} /$ d.o.f. \\
\hline Over-fitted & 1 & 1 & $0.547(6)$ & $0.132(1)$ & $28.059 / 122$ \\
Our choice & 2 & 0 & $0.550(7)$ & $0.0335(3)$ & $31.219 / 121$ \\
\hline
\end{tabular}

\section{A.3. Time discretisation and the calculation of the relaxation function $S\left(t, t_{\mathrm{w}} ; H\right)$}

As explained in the main text, the quantity used in experiment [5] to extract $t^{\text {eff }}(H)$ is the relaxation function $S\left(t, t_{\mathrm{w}} ; H\right)$ of equation $(11)$. We calculate $S\left(t, t_{\mathrm{w}} ; H\right)$ as a finite-time difference:

$$
S\left(t, t_{\mathrm{w}}, t^{\prime} ; H\right)=\frac{M_{\mathrm{ZFC}}\left(t^{\prime}, t_{\mathrm{w}} ; H\right)-M_{\mathrm{ZFC}}\left(t, t_{\mathrm{w}} ; H\right)}{\ln \left(\frac{t^{\prime}}{t}\right)} .
$$

In simulations, time is discrete and we have stored configurations at $t_{n}=$ integer-part-of $2^{n / 4}$, with $n$ an integer. Let us write the integer dependence of times $t$ and $t^{\prime}$ explicitly as:

$$
t \equiv t_{n}, \quad t^{\prime} \equiv t_{n+k},
$$

where $k$ is an integer time parameter. Hence, time is rescaled as

$$
t_{\text {new }}=\frac{1}{2} \ln \left(\frac{t_{n+k}}{t_{n}}\right) \text {. }
$$

We expressed our observables as functions of $t_{\text {new }}$ :

$$
\begin{aligned}
S\left(t, t_{\mathrm{w}}, t^{\prime} ; H\right) & \rightarrow S\left(t_{\mathrm{new}}, t_{\mathrm{w}} ; H\right), \\
C\left(t, t_{\mathrm{w}} ; H\right) & \rightarrow C\left(t_{\text {new }}, t_{\mathrm{w}} ; H\right) .
\end{aligned}
$$


The relaxation function $S\left(t_{\text {new }}, t_{\mathrm{w}} ; H\right)$ is trivial to construct, see equation (A.4). However, the correlation function $C\left(t_{\text {new }}, t_{\mathrm{w}} ; H\right)$ needs to be calculated using a linear interpolation. For any given value of $t_{\text {new }}$, we looked for our original discrete time $t_{n}$ such that

$$
\ln \left(t_{n}\right)<\ln \left(t_{\text {new }}\right) \leqslant \ln \left(t_{n+1}\right) .
$$

Using a linear interpolation, we obtain

$$
C\left(t_{\text {new }}\right)=\frac{\ln \left(t_{\text {new }}\right)-\ln \left(t_{n+1}\right)}{\ln \left(t_{n}\right)-\ln \left(t_{n+1}\right)} C\left(t_{n}\right)-\frac{\ln \left(t_{\text {new }}\right)-\ln \left(t_{n}\right)}{\ln \left(t_{n}\right)-\ln \left(t_{n+1}\right)} C\left(t_{n+1}\right) .
$$

Finally, one can express the relaxation function, $S\left(t_{\mathrm{new}}, t_{\mathrm{w}} ; H\right)$, as a function of the correlation function, $\mathcal{S}(C ; H)$, in much the same manner as equation (A.10).

\section{A.4. The $t_{H}^{\text {eff }}$ calculation}

As explained in the main text, the extraction of $t_{H}^{\text {eff }}$ from equation (16) is delicate because the $C_{\text {peak }}\left(t_{\mathrm{w}}\right)$ are implicit functions of the rescaled time $t_{\text {new }}$. In order to solve equation (16), we calculate the $t_{H}^{\text {eff }}$ values through a quadratic interpolation. First, we calculate the original discrete time $t_{n}$ such that:

$$
C\left(t+t_{n+1}, t_{\mathrm{w}} ; H\right)<C_{\text {peak }} \leqslant C\left(t+t_{n}, t_{\mathrm{w}} ; H\right) .
$$

Then, we solve the three-equation system:

$$
\begin{aligned}
C\left(t+t_{n-1}\right) & =\alpha_{0}+\alpha_{1} x_{n-1}+\alpha_{2} x_{n-1}^{2}, \\
C\left(t+t_{n}\right) & =\alpha_{0}+\alpha_{1} x_{n}+\alpha_{2} x_{n}^{2}, \\
C\left(t+t_{n+1}\right) & =\alpha_{0}+\alpha_{1} x_{n+1}+\alpha_{2} x_{n+1}^{2},
\end{aligned}
$$

where $x_{n}=\ln t_{n}$ and, for brevity's sake, we omit the arguments $t_{\mathrm{w}}$ and $H$. The solution generates the $\alpha_{i}$ coefficients:

$$
\begin{aligned}
\alpha_{2}= & \frac{C\left(t_{\mathrm{w}}, t_{\mathrm{w}}+t_{n-1}\right)-C\left(t_{\mathrm{w}}, t_{\mathrm{w}}+t_{n}\right)}{x_{n-1}-x_{n}} \\
& -\frac{C\left(t_{\mathrm{w}}, t_{\mathrm{w}}+t_{n+1}\right)-C\left(t_{\mathrm{w}}, t_{\mathrm{w}}+t_{n}\right)}{\left(x_{n-1}-x_{n+1}\right)\left(x_{n+1}-x_{n}\right)}, \\
\alpha_{1}= & \frac{C\left(t_{\mathrm{w}}, t_{\mathrm{w}}+t_{n+1}\right)-C\left(t_{\mathrm{w}}, t_{\mathrm{w}}+t_{n}\right)}{x_{n+1}-x_{n}}-\alpha_{2}\left(x_{n}+x_{n+1}\right), \\
\alpha_{0}= & C\left(t_{\mathrm{w}}, t_{\mathrm{w}}+t_{n}\right)-\alpha_{1} x_{n}-\alpha_{2} x_{n}^{2} .
\end{aligned}
$$

We can then calculate the $t_{H}^{\mathrm{eff}}$ solving the equation:

$$
C_{\text {peak }}=\alpha_{0}+\alpha_{1} \ln \left(t_{H}^{\mathrm{eff}}\right)+\alpha_{2}\left[\ln \left(t_{H}^{\mathrm{eff}}\right)\right]^{2},
$$

where only the solution verifying $t_{n} \leqslant t_{H}^{\mathrm{eff}}<t_{n+1}$ is physical. 
Spin-glass dynamics in the presence of a magnetic field: exploration of microscopic properties

\section{A.5. The construction of $\xi\left(t, t_{\mathrm{w}} ; H\right)$}

We shall explain here our computation of the spin-glass correlation length in the presence of a magnetic field. For the simpler case of $H=0$, we refer the reader to $[2,65]$.

The most informative connected correlator we can construct with four replicas is the replicon propagator $[60,66]$. Extending the replicon propagator to the off-equilibrium regime we have:

$$
G_{\mathrm{R}}\left(\boldsymbol{r}, t^{\prime}\right)=\frac{1}{V} \sum_{x} \overline{\left(\left\langle s_{x, t^{\prime}} s_{x+r, t^{\prime}}\right\rangle-\left\langle s_{x, t^{\prime}}\right\rangle\left\langle s_{x+r, t^{\prime}}\right\rangle\right)^{2}},
$$

where $t^{\prime}=t_{\mathrm{w}}+t$. To compute $G_{\mathrm{R}}\left(\boldsymbol{r}, t^{\prime}\right)$, we calculate the four-replica field

$$
\Phi_{x, \mathrm{t}^{\prime}}^{(a, b ; c, d)}=\frac{1}{2}\left(s_{\boldsymbol{x}, \mathrm{t}^{\prime}}^{(a)}-s_{\boldsymbol{x}, \mathrm{t}^{\prime}}^{(b)}\right)\left(s_{\boldsymbol{x}, \mathrm{t}^{\prime}}^{(c)}-s_{\boldsymbol{x}, \mathrm{t}^{\prime}}^{(d)}\right)
$$

where indices $a, b, c, d$ indicate strictly different replica. Notice that

$$
\left\langle\Phi_{x, t^{\prime}}^{(a, b, c, d)} \Phi_{\boldsymbol{y}, \mathrm{t}^{\prime}}^{(a, b ; c, d)}\right\rangle=\left(\left\langle s_{\boldsymbol{x}, \mathrm{t}^{\prime}} \boldsymbol{s}_{\boldsymbol{y}, \mathrm{t}^{\prime}}\right\rangle-\left\langle s_{\boldsymbol{x}, \mathrm{t}^{\prime}}\right\rangle\left\langle s_{\boldsymbol{y}, \mathrm{t}^{\prime}}\right\rangle\right)^{2} .
$$

Therefore, we obtain $G_{\mathrm{R}}\left(\boldsymbol{r}, t^{\prime}\right)$ by taking the average over the samples

$$
\mathrm{E}\left(\Phi_{\boldsymbol{x}, \mathrm{t}^{\prime}}^{(a, b d)} \Phi_{\boldsymbol{y}, \mathrm{t}^{\prime}}^{(a, b ; c, d)}\right)=G_{\mathrm{R}}\left(\boldsymbol{x}-\boldsymbol{y}, t^{\prime}\right) .
$$

With 512 replicas at our disposal, there are $3 \times 512 ! /(508 ! \times 4 !)$ ways of choosing the replica indices $a, b, c$, and $d$. We have found an efficient way for averaging over (roughly) one third of this astronomic number of possibilities [67]. We define the correlation function, $C\left(\boldsymbol{r}, t^{\prime}\right)$, as the replicon propagator, $G_{\mathrm{R}}\left(\boldsymbol{x}-\boldsymbol{y}, t^{\prime}\right)$, where we consider the difference $\boldsymbol{x}-\boldsymbol{y}$ as the lattice displacement $\boldsymbol{r}=(r, 0,0)$. Of course, we can align the lattice displacement vector $\boldsymbol{r}$ along any of three coordinate axes, so we average over these three choices. The replicon correlator $G_{\mathrm{R}}$ decays to zero in the large-distance limit. We, therefore, computed the correlation length $\xi\left(t, t_{\mathrm{w}} ; H\right)$ exploiting the integral estimators $[7,8]$ :

$$
I_{k}\left(t^{\prime} ; T\right)=\int_{0}^{\infty} \mathrm{d} r r^{k} C\left(r, t^{\prime} ; T\right), \quad \xi_{k, k+1}\left(t^{\prime} ; T\right)=\frac{I_{k+1}\left(t^{\prime} ; T\right)}{I_{k}\left(t^{\prime} ; T\right)} .
$$

In the main text, we always refer to the $\xi_{12}\left(t^{\prime} ; T\right)$ correlation length except for section 5.6.2, where we evaluate $\xi_{23}\left(t^{\prime} ; T\right)$.

\section{Appendix B. The Josephson length}

For the reader's convenience, we reproduce here the interpolation proposed in [3] of the data obtained in [2] for the replicon exponent as a function of the Josephson length and the correlation length. 
Spin-glass dynamics in the presence of a magnetic field: exploration of microscopic properties

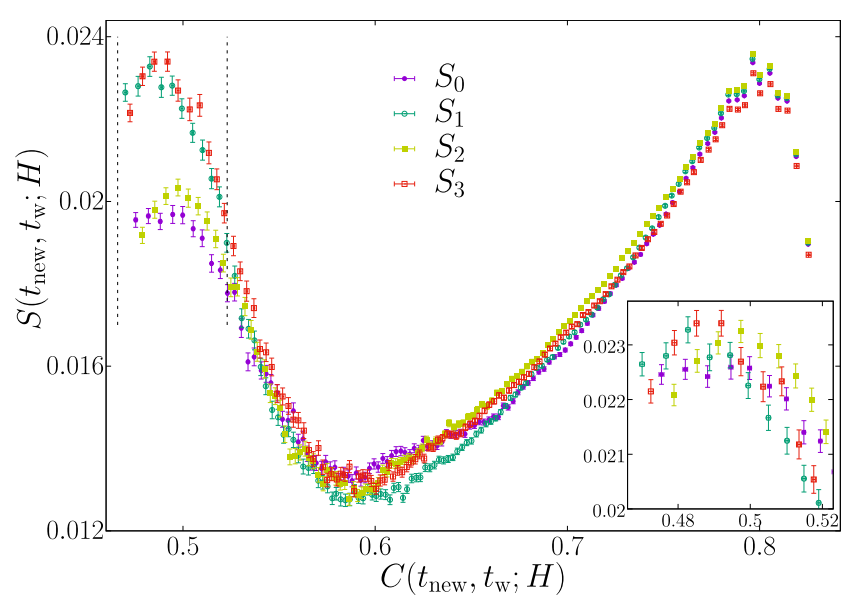

Figure 24. Plots show the behaviour of $\mathcal{S}(C ; H)$ for four independent samples for $H=0.005, t_{\mathrm{w}}=2^{31.25}$ at $T=0.9$. The peak area is enlarged in the inset after a vertical shift.

The Josephson length, $\ell_{\mathrm{J}}\left(T^{(J)}\right)$, scales as

$$
\ell_{\mathrm{J}}\left(T^{(J)}\right)=\frac{b_{0}+b_{1}\left(T_{\mathrm{c}}^{(J)}-T^{(J)}\right)^{\nu}+b_{2}\left(T_{\mathrm{c}}^{(J)}-T^{(J)}\right)^{\omega \nu}}{\left(T_{\mathrm{c}}^{(J)}-T^{(J)}\right)^{\nu}}
$$

where $T^{(J)}$ is the temperature in IEA units,

$$
T^{(J)}=\frac{T}{T_{\mathrm{c}}} T_{\mathrm{c}}^{(J)} \quad T_{\mathrm{c}}^{(J)}=1.102(3),
$$

and we have included analytic $\left(b_{1}\right)$ and confluent $\left(b_{2}\right)$ scaling corrections with $\omega=$ 1.12(10) and $\nu=2.56(4)[27]$. The numerical coefficients are:

$$
\omega=1.12(10), \nu=2.56(4), b_{0} \approx 0.1015, b_{1} \approx 0.3725, b_{2} \approx 0.1997 .
$$

The replicon exponent $\theta(\xi ; T)$ depends on both the temperature and the correlation length $\xi$ through the crossover variable

$$
\tilde{x}=\frac{\ell_{\mathrm{J}}(T)}{\xi\left(t_{\mathrm{w}} ; T\right)} .
$$

In fact, $\theta(\tilde{x}(\xi ; T))$ can be well interpolated as,

$$
\theta(x)=\theta_{0}+d_{1}\left(\frac{x}{1+e_{1} x}\right)^{2-\theta_{0}}+d_{2}\left(\frac{x}{1+e_{2} x}\right)^{3-\theta_{0}},
$$

where

$$
\begin{aligned}
& \theta_{0} \approx 0.30398, \quad e_{1} \approx 1.38179, \quad d_{1} \approx 2.72489, \\
& e_{2} \approx 2.12634, \quad d_{2} \approx-9.98389 .
\end{aligned}
$$


Spin-glass dynamics in the presence of a magnetic field: exploration of microscopic properties

Table 10. Values of $t_{H}^{\mathrm{eff}}$ and $C_{\text {peak }}\left(t_{\mathrm{w}}\right)$ for four independent samples in the case $H=0.005, t_{\mathrm{w}}=2^{31.25}$ and $T=0.9$.

\begin{tabular}{lcc}
\hline Sample & $\log _{2}\left(t_{H}^{\mathrm{eff}}\right)$ & $C_{\text {peak }}\left(t_{\mathrm{w}}\right)$ \\
\hline$S_{0}$ & $30.434(21)$ & 0.493 \\
$S_{1}$ & $30.196(23)$ & 0.493 \\
$S_{2}$ & $30.546(24)$ & 0.505 \\
$S_{3}$ & $30.327(21)$ & 0.505 \\
\hline
\end{tabular}

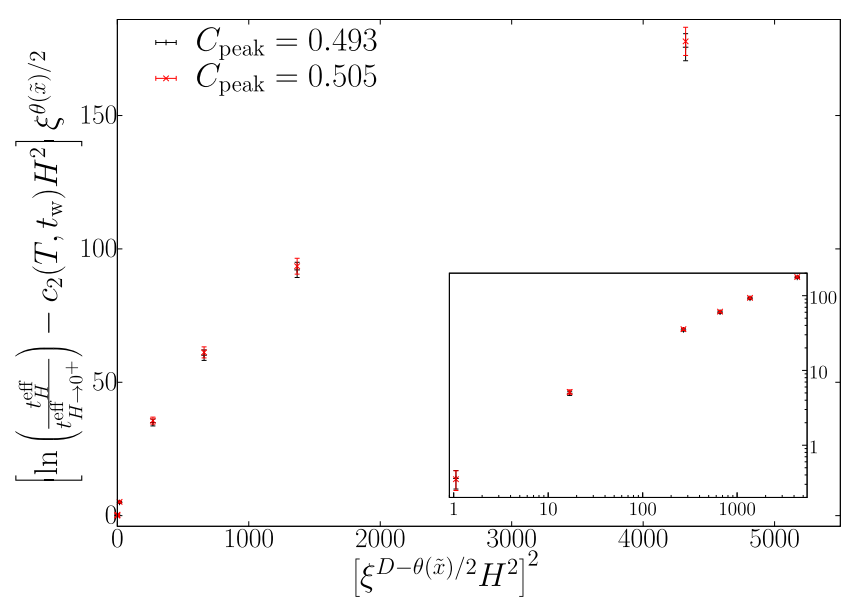

Figure 25. Behaviour of the scaling law for the case $t_{\mathrm{w}}=2^{31.25}$ at $T=0.9$ for the two different $C_{\text {peak }}$ values in table 10. The main figure is in semi-log scale whereas the insert is amplified in a $\log -\log$ scale. It can be seen that the data points for the two values of $C_{\text {peak }}$ are equivalent within their respective error bars.

\section{Appendix C. Sample dependence of the non-linear scaling results}

We demonstrate that the non-linear scaling results are sample independent for the case $H=0.005, t_{\mathrm{w}}=2^{31.25}$, at $T=0.9$. We simulate four independent samples and, for each one, we build the relaxation function $\mathcal{S}(C ; H)$, see section 4.2 and appendix A.3. We exhibit them in figure 24 where we report only the case for $k=8$. To compare peak regions of the relaxation function, $\mathcal{S}(C, H)$, we shift the lowest curves, namely $S_{0}$ and $S_{2}$, vertically. An amplification of the peak region is shown in the inset of figure 24 . As one can observe, there is a sample dependence of the peak position. We report the estimates of $C_{\text {peak }}\left(t_{\mathrm{w}}\right)$ for each sample in table 10 . Note that the sample $S_{0}$ is the one analysed in the main text. We extract the effective time $t_{H}^{\mathrm{eff}}$ for each sample, according to equation (16). They are listed in table 10. The sample dependence found for the $C_{\text {peak }}^{S_{i}}\left(t_{\mathrm{w}}\right)$ values is seen in the $t_{H}^{\mathrm{eff}}$ values too. Accordingly, we repeat the analysis of section 5.4 using, as input parameter for extracting $t_{H}^{\mathrm{eff}}$, the $C_{\text {peak }}^{S_{2}}\left(t_{\mathrm{w}}\right)$ value shown in table 10 . We analyse the effective time ratio $\ln \frac{t_{H}^{\text {eff }}}{t_{H \rightarrow 0^{+}}^{\text {eff }}}$ according to equation $(30)$. We 
then compare the scaling behaviour for the two values of $C_{\text {peak }}^{S_{i}}\left(t_{\mathrm{w}}\right)$. The two sets of data are statistically compatible, see figure 25. This implies that the physical scenario is not affected by the small uncertainty in the determination of $C_{\text {peak }}\left(t_{\mathrm{w}}\right)$. We therefore assert that the scaling results are sample independent.

\section{References}

[1] Cui W, Marsland R and Mehta P 2020 Phys. Rev. Lett. 125048101

[2] Baity-Jesi M et al (Janus Collaboration) 2018 Phys. Rev. Lett. 120267203

[3] Zhai Q, Martin-Mayor V, Schlagel D L, Kenning G G and Orbach R L 2019 Phys. Rev. B 100094202

[4] Zhai Q et al 2020 Phys. Rev. Lett. 125237202

[5] Joh Y G, Orbach R, Wood G G, Hammann J and Vincent E 1999 Phys. Rev. Lett. $82438-41$

[6] Zhai Q, Harrison D C and Orbach R L 2017 Phys. Rev. B 96054408

[7] Belletti F et al (Janus Collaboration) 2008 Phys. Rev. Lett. 101157201

[8] Belletti F et al (Janus Collaboration) 2009 J. Stat. Phys. 1351121

[9] Bert F, Dupuis V, Vincent E, Hammann J and Bouchaud J P 2004 Phys. Rev. Lett. 92167203

[10] Bray A J and Moore M A 1987 Phys. Rev. Lett. 58 57-60

[11] Banavar J R and Bray A J 1987 Phys. Rev. B 358888

[12] Baity-Jesi M et al (Janus Collaboration) 2020 arXiv:2011.09419

[13] Jonason K, Vincent E, Hammann J, Bouchaud J P and Nordblad P 1998 Phys. Rev. Lett. 81 3243-6

[14] Nordblad P and Svedlindh P 1997 Experiments on spin glasses Spin Glasses and Random Fields (Directions in Condensed Matter Physics) vol 12 ed A P Young (Singapore: World Scientific) p 1

[15] Vincent E, Hammann J, Ocio M, Bouchaud J P and Cugliandolo L F 1997 Slow dynamics and aging in spin glasses Complex Behavior of Glassy Systems (Lecture Notes in Physics No 492) ed M Rubí and C Pérez-Vicente (Berlin: Springer)

[16] Vincent E 2007 Ageing, rejuvenation and memory: the example of spin glasses Ageing and the Glass Transition (Lecture Notes in Physics No 716) ed M Henkel, M Pleimling and R Sanctuary (Berlin: Springer)

[17] Nordblad P, Svedlindh P, Lundgren L and Sandlund L 1986 Phys. Rev. B 33 645-8

[18] Lederman M, Orbach R, Hammann J M, Ocio M and Vincent E 1991 Phys. Rev. B 44 7403-12

[19] Hammann J, Lederman M, Ocio M, Orbach R and Vincent E 1992 Physica A 185278

[20] Guchhait S and Orbach R 2014 Phys. Rev. Lett. 112126401

[21] Bouchaud J P 1992 J. Phys. I France 2 1705-13

[22] Vincent E, Bouchaud J P, Dean D S and Hammann J 1995 Phys. Rev. B 52 1050-60

[23] Baity-Jesi M et al (Janus Collaboration) 2017 Phys. Rev. Lett. 118157202

[24] Rodriguez G F 2004 PhD Dissertation University of California, Riverside (https://researchgate.net/publication/ 252531893_Initial_conditions_and_long_time_dynamics_for_a_complex_system_Hierarchical_properties_of_the_ spin_glass_decay)

[25] Rodriguez G, Kenning G and Orbach R 2013 Phys. Rev. B 88054302

[26] Baity-Jesi M et al (Janus Collaboration) 2014 Comput. Phys. Commun. 185 550-9

[27] Baity-Jesi M et al (Janus Collaboration) 2013 Phys. Rev. B 88224416

[28] Mydosh J A 1993 Spin Glasses: An Experimental Introduction (London: Taylor and Francis)

[29] Aruga Katori H and Ito A 1994 J. Phys. Soc. Japan 63 3122-8

[30] Mézard M, Parisi G and Virasoro M 1987 Spin-Glass Theory and Beyond (Singapore: World Scientific)

[31] Fisher D S and Sompolinsky H 1985 Phys. Rev. Lett. 541063

[32] Cugliandolo L F and Kurchan J 1993 Phys. Rev. Lett. 71 173-6

[33] Cruz A, Fernández L A, Jiménez S, Ruiz-Lorenzo J J and Tarancón A 2003 Phys. Rev. B 67214425

[34] Franz S, Parisi G and Virasoro M A 1994 J. Phys. I France 41657

[35] Franz S, Mézard M, Parisi G and Peliti L 1998 Phys. Rev. Lett. 81 1758-61

[36] Franz S, Mézard M, Parisi G and Peliti L 1999 J. Stat. Phys. 97 459-88

[37] Malozemoff A P, Barbara B and Imry Y 1982 J. Appl. Phys. 532205

[38] Malozemoff A P, Imry Y and Barbara B 1982 J. Appl. Phys. 537672

[39] Chandra P, Coleman P and Ritchey I 1993 J. Phys. I France 3 591-610

[40] Lévy L P and Ogielski A T 1986 Phys. Rev. Lett. 57 3288-91

[41] Lévy L P 1988 Phys. Rev. B 38 4963-73 
Spin-glass dynamics in the presence of a magnetic field: exploration of microscopic properties

[42] Parisi G 1988 Statistical Field Theory (Reading, MA: Addison-Wesley)

[43] Amit D J and Martín-Mayor V 2005 Field Theory, the Renormalization Group and Critical Phenomena 3rd edn (Singapore: World Scientific) (http://worldscientific.com/worldscibooks/10.1142/5715)

[44] Rodriguez G F, Kenning G G and Orbach R 2003 Phys. Rev. Lett. 91037203

[45] Baity-Jesi M et al 2017 Proc. Natl Acad. Sci. 114 1838-43

[46] Kisker J, Santen L, Schreckenberg M and Rieger H 1996 Phys. Rev. B 53 6418-28

[47] Zhai Q, Orbach R L and Schlagel D 2020 arXiv:2010.01214

[48] Bouchaud J-P, Doussineau P, de Lacerda-Arôso T and Levelut A 2001 Eur. Phys. J. B 21335

[49] Simmons-Duffin D 2017 J. High Energy Phys. 8688

[50] Hasenbusch M 2020 Phys. Rev. E 101022126

[51] Ferrenberg A M, Xu J and Landau D P 2018 Phys. Rev. E 97043301

[52] Campostrini M, Pelissetto A, Rossi P and Vicari E 2002 Phys. Rev. E 65066127

[53] Hasenbusch M and Vicari E 2011 Phys. Rev. B 84125136

[54] Astillero A and Ruiz-Lorenzo J J 2019 Phys. Rev. E 100062117

[55] Ballesteros H G, Fernández L A, Martín-Mayor V and Muñoz Sudupe A 1996 Phys. Lett. B 387125

[56] Baños R A et al 2012 Proc. Natl Acad. Sci. 1096452

[57] Larson D, Katzgraber H G, Moore M A and Young A P 2013 Phys. Rev. B 87024414

[58] Baity-Jesi M et al 2014 J. Stat. Mech. P05014

[59] Höller J and Read N 2020 Phys. Rev. E 101042114

[60] de Almeida J R L and Thouless D J 1978 J. Phys. A: Math. Gen. 11983

[61] Sherrington D 1978 J. Phys. A: Math. Gen. 11 L185

[62] Fisher D S and Huse D A 1986 Phys. Rev. Lett. 561601

[63] Bray A J, Moore M A and Young A P 1986 Phys. Rev. Lett. 56 2641-4

[64] Lefloch F, Hammann J, Ocio M and Vincent E 1994 Physica B 203 63-74

[65] Fernández L A, Marinari E, Martín-Mayor V, Parisi G and Ruiz-Lorenzo J J 2019 J. Phys. A: Math. Theor. 52 224002

[66] de Dominicis C and Giardina I 2006 Random Fields and Spin Glasses: A Field Theory Approach (Cambridge: Cambridge University Press)

[67] Paga I 2020 PhD Thesis La Sapienza, Rome and Universidad Complutense de Madrid in preparation 